IOS Press

\title{
Isokinetic testing of muscle strength in older adults with knee osteoarthritis: An integrative review
}

\author{
Bradley J. Myers \\ Department of Physical Therapy, College of Pharmacy and Health Sciences, Campbell University, Lillington, \\ NC 27546, USA \\ Tel.: +1910814 8043; E-mail: bmyers@campbell.edu
}

\begin{abstract}
.
BACKGROUND: Knee osteoarthritis (OA) is common among older adults and associated with impaired knee strength. OBJECTIVE: Describe isokinetic testing procedures and clinimetric findings associated with the testing of knee strength in the presence of knee OA.

METHODS: Relevant articles were identified by an electronic search of PubMed using the search string "isokinet* AND knee osteoarthritis."

RESULTS: One-hundred and twenty-nine relevant articles were found. The articles support the validity and reliability of isokinetic strength testing for patients with knee OA. The responsiveness to various therapeutic interventions has been reported.

CONCLUSIONS: Isokinetic dynamometry is a valid and reliable measure of muscle strength in knee OA.
\end{abstract}

Keywords: Muscle strength, measurement, knee osteoarthritis

\section{Introduction}

Knee osteoarthritis (OA) is a common musculoskeletal condition that is responsible for approximately $80 \%$ of the global burden related to osteoarthritis at all sites within the body [1]. The individual and societal burden of knee OA is negatively associated with physical activity, disability, morbidity, and mortality levels [2,3]. Muscle strength has been implicated in disease severity and highlighted as a target for therapeutic rehabilitation [4-7]. There are several options for assessing lower extremity muscle strength in the presence of knee OA including manual muscle testing, hand-held dynamometry, and isokinetic dynamometry. The purpose of this review was to highlight procedures and results of isokinetic testing utilized for the measurement of knee strength in the presence of knee OA.

\section{Methods}

PubMed was searched for articles potentially related to isokinetic testing and knee arthritis on July 7, 2019. The search string included "isokinet* AND knee osteoarthritis." Article titles and abstracts were initially reviewed for relevance and if warranted the full text was then examined to determine if the articles addressed isokinetic strength testing for individuals with knee OA. Articles were excluded if published in a language other than English, were review articles, failed to provide procedural specifics or presented no findings relevant to the clinimetric properties of isokinetic testing. Articles retained for this review were mined for information on the sample tested, procedural specifics (e.g., speed and movement tested), and clinically relevant findings.

\section{Results and discussion}

The PubMed search identified 312 potentially relevant articles. After review, 183 articles were excluded leaving 129 articles for inclusion in this report. Rele- 
Table 1

Summary of studies describing the isokinetic measurement of strength in knee osteoarthritis (OA)

\begin{tabular}{|c|c|c|c|}
\hline $\begin{array}{l}\text { Authorship } \\
\text { (year) }\end{array}$ & Participants & Isokinetic procedures & Findings \\
\hline $\begin{array}{l}\text { Aily } \\
(2019)[8]\end{array}$ & $\begin{array}{l}\text { Brazilians with knee OA }(n \\
=40 ; \text { mean age }=59.8 \mathrm{y}) \& \\
\text { without knee OA }(n=40 ; \\
\text { mean age }=59.9 \mathrm{y}) .\end{array}$ & $\begin{array}{l}\text { Biodex System } 3 \text { Pro dynamometer } \\
\text { measured knee extensor strength (PT } \\
\& \text { body weight normalized PT) @ } \\
60^{\circ} / \mathrm{s} \& \text { isometric extensor strength } \\
\text { (PT \& body weight normalized PT). }\end{array}$ & $\begin{array}{l}\text { Validity: knee extensor strength in middle-aged without } \\
\text { knee OA SGNF > older-aged without knee OA. Knee } \\
\text { extensor strength in middle-aged with knee OA SGNF > } \\
\text { older-aged with knee OA. No difference in knee extensor } \\
\text { strength between middle-aged with knee OA \& } \\
\text { older-aged without knee OA. }\end{array}$ \\
\hline $\begin{array}{l}\text { Akyol et al. } \\
\text { (2010) [9] }\end{array}$ & $\begin{array}{l}\text { Turkish females with } \\
\text { bilateral knee OA assigned } \\
\text { to isokinetic strength training } \\
\text { with short-wave diathermy } \\
(n=20 ; \text { mean age }= \\
57.8 \text { y) or isokinetic strength }\end{array}$ & $\begin{array}{l}\text { Cybex Human Norm dynamometer } \\
\text { measured concentric flexor \& } \\
\text { extensor strength (PT) @ 60,120, \& } \\
180^{\circ} / \mathrm{s} \text {. }\end{array}$ & $\begin{array}{l}\text { Responsiveness: no difference in knee strength between } \\
\text { groups at } 4 \text { weeks. 3-month improvements in right knee } \\
\text { flexor strength @ } 60 \% \text { s \& right knee extensor strength @ } \\
60 \& 120 \% \text { s for group receiving short-wave diathermy } \\
\text { SGNF }>\text { group without short-wave diathermy. No } \\
\text { between group difference in left limb or other speeds. }\end{array}$ \\
\hline
\end{tabular}

training without short-wave

diathermy $(n=20$; mean age $=56.6 \mathrm{y})$.

Alencar et al. Brazilians with knee OA

(2007) [10] with a history of falling $(n$

Biodex System 3 measured concentric Validity: No difference in concentric knee flexor or $=15$; mean age $=74.2 \mathrm{y}$ ) or weight adjusted PT, work, \& power) without a history of falling @ 60,120, \& $180^{\circ}$ /s.

$(n=5 ;$ mean age $=71.7 \mathrm{y})$.

Alkatan et al. Americans with knee OA Biodex dynamometer measured knee

(2015) [11] assigned to receive cycling flexor \& extensor strength (PT) @ 60 $(n=24$; mean age $=61 \mathrm{y}) \quad \& 120^{\circ} / \mathrm{s}$ or swimming $(n=24$; mean age $=59 \mathrm{y})$.

Almosnino et Canadians with knee OA ( $n$ al. (2012) [12] $=35$; mean age $=62.1 \mathrm{y})$.

Biodex System 3 dynamometer measured concentric knee extensor strength (bodyweight adjusted PT) @ $60,90, \& 120^{\circ} \%$ s.

Altubasi et al. Jordanians with knee OA ( $n$ Biodex System 3 measured isokinetic (2018) $[13]=160$; mean age =63.6 y). knee extensor strength $(\mathrm{PT}) @ 60^{\circ}$.

Amin et al. Americans with knee OA ( $n$ Cybex dynamometer measured (2009) $[14]=265$; mean age $=67 \mathrm{y})$. isokinetic knee extensor strength (bodyweight adjusted PT) @ 60\%

An et al. Chinese females with knee (2008) [15] OA assigned to Baduanjintraditional Chinese exercise $(n=14 ;$ mean age $=$ $65.4 \mathrm{y})$ or control $(n=14$; mean age $=64.6 \mathrm{y})$.

An et al. Chinese with knee OA ( $n=$

(2013) $[16] \quad 22$; mean age $=65.2 \mathrm{y}$ ) assigned to Baduanjin exercise.

Anandkumar Indians with knee OA

(2014) [17] assigned to receive

kinesiotape with tension ( $n$

$=20$; mean age $=55.7 \mathrm{y}$ ) or

kinesiotape without $(n=20$;

mean age $=55.9$ y) tension.
Biodex System 3 Pro dynamometer measured knee flexor \& extensor strength (PT) @60\%.

Cybex Humac NORM dynamometer measured concentric \& eccentric knee extensor strength (PT) @ 90 \& $120 \%$ s.

extensor strength between groups.

Reliability: test-retest reliability in knee strength measurement (ICC $=0.88$ to 0.97 ).

Responsiveness: SGNF $\uparrow$ knee flexor \& extensor strength in both groups.

Validity: concentric knee extensor strength @ $60^{\circ}$ SGNF $>@ 90 \& 120^{\circ}$ s. No difference in pain between test speeds.

Validity: height, weight, age, gender, \& isometric knee extensor strength partially explained variability in Get Up and Go Test $\left(r^{2}=0.41\right)$ and WOMAC physical function subscale $\left(r^{2}=0.21\right)$.

Reliability: test-retest knee extensor strength (ICC = 0.96).

Responsiveness: no relationship between knee extensor strength \& cartilage loss. Highest tertile of knee extensor strength had SGNF $\downarrow$ risk of patellofemoral cartilage loss $(\mathrm{OR}=0.4)$, better WOMAC physical function scores, \& less pain.

Biodex System 3 measured isokinetic Responsiveness: SGNF $\uparrow$ knee extensor strength for knee extensor strength (PT) @60\%.

Responsiveness: SGNF $\uparrow$ in knee flexor \& extensor strength.

Reliability: test-retest concentric knee extensor strength $\overline{(I C C}=0.87$ to $0.92 ; \mathrm{MDC}=0.03$ to $0.05 \mathrm{Nm} / \mathrm{kg}$ ) \& eccentric knee extensor strength (ICC $=0.72$ to 0.86 ; $\mathrm{MDC}=0.14$ to $0.19 \mathrm{Nm} / \mathrm{kg}$ ).

Responsiveness: concentric knee extensor strength in

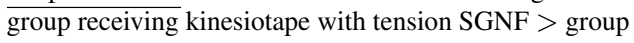
without tension, no between group difference in eccentric knee extensor strength. 
Table 1, continued

\begin{tabular}{|c|c|c|c|}
\hline $\begin{array}{l}\text { Authorship } \\
\text { (year) }\end{array}$ & Participants & Isokinetic procedures & Findings \\
\hline $\begin{array}{l}\text { Bacon et al. } \\
(2018)[18]\end{array}$ & $\begin{array}{l}\text { Americans with knee OA }(n \\
=834 ; \text { mean age }=62.9 \mathrm{y})\end{array}$ & $\begin{array}{l}\text { Cybex } 350 \text { dynamometer measured } \\
\text { knee extensor strength (PT \& } \\
\text { bodyweight adjusted PT) @ } 60^{\circ} / \mathrm{s} \text {. }\end{array}$ & $\begin{array}{l}\text { Validity: knee extensor strength females } \mathrm{SGNF}<\text { males. } \\
\text { Females with knee extensor strength }<38 \mathrm{Nm} \text { was } \\
\text { associated with larger } \uparrow \text { difficulty rising from a chair } \& \\
\text { getting off toilet. Females with knee extensor strength }< \\
0.74 \mathrm{Nm} / \mathrm{kg} \text { associated with larger } \uparrow 20 \mathrm{~m} \text { walk test time. }\end{array}$ \\
\hline $\begin{array}{l}\text { Baert et al. } \\
(2013)[19]\end{array}$ & $\begin{array}{l}\text { Belgians with established } \\
\text { knee OA }(n=24 ; \text { mean age } \\
=64.0 \mathrm{y}) \text {, early knee OA ( } n \\
=21 ; \text { mean age }=65.5 \mathrm{y}) \text { or } \\
\text { healthy controls }(n=20 ; \\
\text { mean age }=62.9 \mathrm{y}) .\end{array}$ & $\begin{array}{l}\text { Biodex System } 3 \text { Pro dynamometer } \\
\text { measured knee flexor \& extensor } \\
\text { strength (body weight adjusted PT) @ } \\
60 \& 240^{\circ} / \mathrm{s} \& \text { isometric knee flexor } \\
\& \text { extensor strength (body weight } \\
\text { adjusted PT) @ } 60 \& 90^{\circ} \text {. }\end{array}$ & $\begin{array}{l}\text { Validity: knee extensor strength in OA groups was SGNF } \\
<\text { healthy group. Knee flexor strength in established OA } \\
\text { group SGNF }<\text { early knee OA or healthy groups. } \\
\text { Reliability: test-retest strength (ICC }=0.75 \text { to } 0.98 \text { ). }\end{array}$ \\
\hline $\begin{array}{l}\text { Baert et al. } \\
(2014)[20]\end{array}$ & $\begin{array}{l}\text { Belgian females (mean age } \\
=65.2 \text { y) with knee OA }(n \\
=45) \& \text { asymptomatic } \\
\text { controls }(n=42)\end{array}$ & $\begin{array}{l}\text { Biodex System } 3 \text { Pro dynamometer } \\
\text { measured knee extensor strength } \\
\text { (body weight adjusted PT) @ } 60 \& \text { \& } \\
240^{\circ} / \mathrm{s} \& \text { isometric knee flexor \& } \\
\text { extensor strength (body weight } \\
\text { adjusted PT) @ } 60 \& 90^{\circ} .\end{array}$ & $\begin{array}{l}\text { Validity: High correlation between all strength mea- } \\
\text { sures }(r>0.70) \text {. SGNF association between Kellgren- } \\
\text { Lawrence grade \& isokinetic extensor strength }(\beta= \\
-0.331) \text { but not isometric flexor or extensor strength. } \\
\text { Variability in isometric extensor strength was partially ex- } \\
\left.\text { plained ( } r^{2}=0.28\right) \text { by patellofemoral cartilage integrity } \\
\& \text { pain. Variability in isokinetic extensor strength was } \\
\left.\text { partially explained ( } r^{2}=0.38\right) \text { by amount of cartilage } \\
\text { lesions, loose bodies \& pain. Variability in isometric knee } \\
\text { flexor strength was partially explained }\left(r^{2}=0.34\right) \text { by } \\
\text { synovitis/effusion, patellofemoral cartilage integrity \& } \\
\text { pain. } \\
\text { Reliability: test-retest all strength measures (ICC }=0.75 \\
\text { to } 0.98) \text {. }\end{array}$ \\
\hline $\begin{array}{l}\text { Baert et al. } \\
(2017)[21]\end{array}$ & $\begin{array}{l}\text { Belgian females with knee } \\
\text { OA }(n=68 ; \text { mean age }= \\
65.6 \text { y) \& healthy controls }(n \\
=56 ; \text { mean age }=65.0 \mathrm{y})\end{array}$ & $\begin{array}{l}\text { Biodex System } 3 \text { measured isometric } \\
\text { flexor \& extensor strength } \\
\text { (bodyweight adjusted PT) @ } 60 \& \\
90^{\circ}, \& \text { isokinetic extensor } \\
\text { strength(bodyweight adjusted PT) @ } \\
60 \& 240^{\circ} / \mathrm{s} \text {. }\end{array}$ & $\begin{array}{l}\text { Validity: knee strength (all measures) in knee OA SGNF } \\
<\text { control group. SGNF inverse correlation of pain } \\
\text { catastrophizing with isometric flexor strength @ } 60 \& \\
90^{\circ}(r=-0.31 \text { to }-0.26) \text {. SGNF inverse correlation of } \\
\text { kinesiophobia \& isometric extensor strength @ } 90^{\circ}(r= \\
-0.26) \text {. No relationship between psychological factors } \\
\text { \& knee strength. }\end{array}$ \\
\hline $\begin{array}{l}\text { Barker } \\
(2018)[22]\end{array}$ & $\begin{array}{l}\text { Americans with knee OA }(n \\
=29 ; \text { mean age }=49 \mathrm{y})\end{array}$ & $\begin{array}{l}\text { Biodex System } 4 \text { dynamometer } \\
\text { measured concentric flexor \& } \\
\text { extensor strength (bodyweight } \\
\text { adjusted PT) @ } 60^{\circ} / \mathrm{s} \& \text { isometric } \\
\text { extension strength (PT) @ } 60^{\circ} .\end{array}$ & $\begin{array}{l}\text { Validity: SGNF correlation of knee strength on limb with } \\
\text { knee OA \& superoxide dismutase but not cytokines. }\end{array}$ \\
\hline $\begin{array}{l}\text { Batista et al. } \\
(2018)[23]\end{array}$ & $\begin{array}{l}\text { Brazilian females with knee } \\
\text { OA }(n=11 ; \text { median age }= \\
62 \text { y) \& healthy controls }(n \\
=10 ; \text { median age }=65 \mathrm{y})\end{array}$ & $\begin{array}{l}\text { Biodex System } 4 \text { measured knee } \\
\text { flexor \& extensor strength } \\
\text { (bodyweight adjusted PT) @ } 90 \text { \& } \\
240^{\circ} / \text { s. }\end{array}$ & $\begin{array}{l}\text { Validity: OA group knee extensor strength @ } 90 \text { \& } \\
240^{\circ} / \mathrm{s}, \& \text { flexor strength @ } 90^{\circ} / \mathrm{s} \text { SGNF }<\text { control } \\
\text { group. }\end{array}$ \\
\hline $\begin{array}{l}\text { Bayramoğlu } \\
\text { et al. } \\
(2003)[24]\end{array}$ & $\begin{array}{l}\text { Turks with knee OA } \\
\text { assigned to physiotherapy ( } n \\
=15 \text {; mean age }=60.7 \mathrm{y}), \\
\text { physiotherapy with low } \\
\text { molecular weight hyaluronic } \\
\text { acid ( } n=15 \text {; mean age }= \\
61.5 \mathrm{y}) \text {, or physiotherapy } \\
\text { with higher molecular } \\
\text { weight hyaluronic acid ( } n= \\
16 \text {; mean age }=62.6 \mathrm{y}) .\end{array}$ & $\begin{array}{l}\text { Cybex } 770 \text { Norm dynamometer } \\
\text { measured flexor \& extensor strength } \\
\text { (PT) @ } 60 \& 90^{\circ} / \mathrm{s} \text {. }\end{array}$ & $\begin{array}{l}\text { Responsiveness: no difference in isokinetic strength } \\
\text { within or between groups over time. }\end{array}$ \\
\hline $\begin{array}{l}\text { Bayramoglu } \\
\text { et al. } \\
(2007)[25]\end{array}$ & $\begin{array}{l}\text { Turks with bilateral knee OA } \\
(n=50 ; \text { mean age }=60.2 \mathrm{y}) \\
\& \text { age matched controls }(n \\
=30 ; \text { mean age }=57.9 \mathrm{y})\end{array}$ & $\begin{array}{l}\text { Cybex } 770 \text { Norm dynamometer } \\
\text { measured isometric flexor \& extensor } \\
\text { strength (PT) @ } 90^{\circ} \text {. }\end{array}$ & $\begin{array}{l}\text { Validity: OA group isometric knee flexor \& extensor } \\
\text { strength SGNF < control group. }\end{array}$ \\
\hline $\begin{array}{l}\text { Bily } \\
(2019)[26]\end{array}$ & $\begin{array}{l}\text { Austrians with knee OA }(n \\
=75 ; \text { mean age }=67.5 \mathrm{y})\end{array}$ & $\begin{array}{l}\text { S2P dynamometer measured } \\
\text { isometric knee extensor strength (PT) } \\
\text { @ } 60^{\circ} \text {. }\end{array}$ & $\begin{array}{l}\text { Validity: SGNF inverse correlation of isometric knee } \\
\text { extension strength with Timed Up and Go Test }(r= \\
-0.41 \text { to }-0.39) \& \text { stair climb test }(r=-0.46) \text {. }\end{array}$ \\
\hline
\end{tabular}


Table 1, continued

\begin{tabular}{|c|c|c|c|}
\hline $\begin{array}{l}\text { Authorship } \\
\text { (year) }\end{array}$ & Participants & Isokinetic procedures & Findings \\
\hline $\begin{array}{l}\text { Bokaeian et } \\
\text { al. } \\
\text { (2016) [27] }\end{array}$ & $\begin{array}{l}\text { Iranians with knee OA } \\
\text { assigned to strength training } \\
\text { with whole body vibration } \\
(n=15 ; \text { mean age }=51.8 \mathrm{y}) \\
\text { or strength training without } \\
\text { whole body vibration }(n= \\
13 ; \text { mean age }=54.0 \mathrm{y}) .\end{array}$ & $\begin{array}{l}\text { Biodex System } 4 \text { Pro dynamometer } \\
\text { measured concentric knee flexor \& } \\
\text { extensor strength (PT, power, \& } \\
\text { work) @ } 90 \& 120^{\circ} / \mathrm{s} \text {. }\end{array}$ & $\begin{array}{l}\text { Responsiveness: } \uparrow \text { knee extensor strength in group } \\
\text { receiving whole body vibration training SGNF }>\text { group } \\
\text { without whole body vibration. }\end{array}$ \\
\hline $\begin{array}{l}\text { Börjesson et } \\
\text { al. } \\
\text { (1996) [28] }\end{array}$ & $\begin{array}{l}\text { Swedes with knee OA } \\
\text { assigned to physiotherapy ( } n \\
=34 ; \text { mean age }=64 \text { y) or } \\
\text { control }(n=37 ; \text { mean age } \\
=64 \mathrm{y})\end{array}$ & $\begin{array}{l}\text { Cybex II dynamometer measured } \\
\text { isokinetic flexor \& extensor strength } \\
\text { (PT) at } 30^{\circ} / \mathrm{s} \text {. }\end{array}$ & $\begin{array}{l}\text { Responsiveness: no difference in knee extensor or flexor } \\
\text { strength after physiotherapy compared to control. }\end{array}$ \\
\hline $\begin{array}{l}\text { Brandt et al. } \\
\text { (1999) [29] }\end{array}$ & $\begin{array}{l}\text { Americans with knee OA }(n \\
=79 ; \text { mean age }=71.6 \mathrm{y})\end{array}$ & $\begin{array}{l}\text { KIN-COM 500H dynamometer } \\
\text { measured knee flexor \& extensor } \\
\text { strength (body weight adjusted PT) @ } \\
60 \& 120^{\circ} / \mathrm{s} \text {. }\end{array}$ & $\begin{array}{l}\text { Validity: no difference in knee flexor \& extensor strength } \\
\text { between females with progressive or stable knee OA. } \\
\text { Body weight adjusted knee extensor strength was in- } \\
\text { versely correlated with body weight }(r=-0.60 \text { to } \\
-0.39) \text { in females. } \\
\text { Reliability: test-retest knee strength (ICC }=0.90) \text {. }\end{array}$ \\
\hline $\begin{array}{l}\text { Brandt et al. } \\
(2000)[30]\end{array}$ & $\begin{array}{l}\text { Americans with radiographic } \\
\text { knee } \mathrm{OA}(n=35 ; \text { mean age } \\
=71.7 \mathrm{y}) \& \text { without } \\
\text { radiographic knee } \mathrm{OA}(n= \\
171 ; \text { median age }=70.3 \mathrm{y})\end{array}$ & $\begin{array}{l}\text { KIN-COM 500H dynamometer } \\
\text { measured knee flexor \& extensor } \\
\text { strength (PT \& bodyweight adjusted } \\
\text { PT) @ } 60^{\circ} \% \text { s. }\end{array}$ & $\begin{array}{l}\text { Validity: knee extensor strength with knee OA SGNF }< \\
\text { without knee OA. Knee extensor strength with knee pain } \\
\text { without radiographic OA SGNF }<\text { healthy controls. No } \\
\text { difference in knee extensor strength between females } \\
\text { with knee OA \& females without radiographic knee OA } \\
\text { but with knee pain. Knee flexor strength in females with } \\
\text { knee pain without radiographic knee OA SGNF }< \\
\text { compared to those with knee OA or controls. In females } \\
\text { with knee pain, individuals with radiographic knee OA } \\
\text { had SGNF } \downarrow \text { flexor strength than those without } \\
\text { radiographic knee OA. No difference in flexor strength } \\
\text { between radiographic knee OA \& controls for knee } \\
\text { flexor strength. }\end{array}$ \\
\hline $\begin{array}{l}\text { Bülow et al. } \\
\text { (1994) [31] }\end{array}$ & $\begin{array}{l}\text { Danes with knee OA } \\
\text { (median age }=74 \text { y) } \\
\text { assigned to low power laser } \\
(n=14) \text { or placebo }(n=7) .\end{array}$ & $\begin{array}{l}\text { Kin-Com dynamometer measured } \\
\text { knee extensor strength (PT) @ 60\%. }\end{array}$ & $\begin{array}{l}\text { Responsiveness: no between group differences in knee } \\
\text { extensor strength. }\end{array}$ \\
\hline $\begin{array}{l}\text { Carpenter et } \\
\text { al. } \\
(2006)[32]\end{array}$ & $\begin{array}{l}\text { Americans with mild knee } \\
\text { OA }(n=18 ; \text { mean age }= \\
54.1 \mathrm{y}) .\end{array}$ & $\begin{array}{l}\text { Biodex Multi-joint System B2000 } \\
\text { measured extensor \& flexor isokinetic } \\
\text { strength (bodyweight adjusted PT, } \\
\text { work, power, \& fatigue) @ } 60 \text { \& } \\
180^{\circ} / \mathrm{s}, \& \text { isometric extensor strength } \\
\text { (maximum average torque, maximum } \\
\text { repetition work)@ } 30,45, \& 80^{\circ} .\end{array}$ & $\begin{array}{l}\text { Reliability: test-retest isometric strength }(\mathrm{ICC}=0.81 \text { to } \\
0.96) \& \text { isokinetic strength (ICC }=0.60 \text { to } 0.95) .\end{array}$ \\
\hline $\begin{array}{l}\text { Cavanellas et } \\
\text { al. } \\
\text { (2018) [33] }\end{array}$ & $\begin{array}{l}\text { Brazilians with mild knee } \\
\text { OA }(n=20 ; \text { mean age }= \\
56.3 \mathrm{y}), \text { severe knee OA }(n \\
=20 ; \text { mean age }=66.1 \mathrm{y}), \\
6 \text { months post TKA }(n= \\
20 ; \text { mean age }=69.1 \mathrm{y}), \\
12 \text { months post TKA }(n= \\
20 ; \text { mean age }=65.8 \mathrm{y}), \& \\
\text { healthy controls }(n=20 ; \\
\text { men age }=67.9 \mathrm{y}) .\end{array}$ & $\begin{array}{l}\text { CSMI dynamometer measured } \\
\text { maximal knee flexion \& extension } \\
\text { strength (bodyweight adjusted PT) @ } \\
60^{\circ} / \mathrm{s} \text {. }\end{array}$ & $\begin{array}{l}\text { Validity: severe knee OA or post-TKA knee extensor \& } \\
\text { flexor strength SGNF }<\text { control or mild knee OA groups. }\end{array}$ \\
\hline
\end{tabular}


Table 1, continued

\begin{tabular}{|c|c|c|c|}
\hline $\begin{array}{l}\text { Authorship } \\
\text { (year) }\end{array}$ & Participants & Isokinetic procedures & Findings \\
\hline $\begin{array}{l}\text { Cetin et al. } \\
(2008)[34]\end{array}$ & $\begin{array}{l}\text { Turks with bilateral knee OA } \\
\text { assigned to isokinetic } \\
\text { exercise with receive short- } \\
\text { wave diathermy \& hot packs } \\
(n=20 ; \text { mean age }= \\
59.8 \text { y), isokinetic exercise } \\
\text { with transcutaneous electric } \\
\text { stimulation \& hot packs ( } n \\
=20 ; \text { mean age }=61.9 \text { y), } \\
\text { isokinetic exercise with } \\
\text { ultrasound \& hot packs ( } n= \\
20 ; \text { mean age }=57.6 \text { y), } \\
\text { isokinetic exercise with hot } \\
\text { packs }(n=20 ; \text { mean age }= \\
61.1 \text { y), or isokinetic } \\
\text { exercise only ( } n=20 \text {; mean } \\
\text { age }=58.9 \text { y). }\end{array}$ & $\begin{array}{l}\text { Cybex } 770 \text { Norm measured } \\
\text { concentric knee flexor \& extensor } \\
\text { strength }(\mathrm{PT}) @ 60,120, \& 180^{\circ} / \mathrm{s} \text {. }\end{array}$ & $\begin{array}{l}\text { Responsiveness: knee extensor strength SGNF } \uparrow \text { in all } \\
\text { groups, with strength } \uparrow \text { in groups receiving short-wave } \\
\text { diathermy \& hot packs, transcutaneous electric } \\
\text { stimulation \& hot packs, or ultrasound \& hot packs }> \\
\text { control group. Knee flexor strength } \uparrow \text { for all groups } \\
\text { SGNF > control group. }\end{array}$ \\
\hline $\begin{array}{l}\text { Chen et al. } \\
(2008)[35]\end{array}$ & $\begin{array}{l}\text { Taiwanese with mild to } \\
\text { moderate knee OA assigned } \\
\text { to wear magnetic knee wrap } \\
(n=21 ; \text { mean age }= \\
64.2 \text { y) or sham wrap }(n= \\
21 ; \text { mean age }=66.1 \mathrm{y})\end{array}$ & $\begin{array}{l}\text { Biodex System } 3 \text { measured concentric } \\
\text { knee extensor strength (bodyweight } \\
\text { adjusted PT) @ } 30 \& 60^{\circ} \text { s. }\end{array}$ & $\begin{array}{l}\text { Responsiveness: knee extensor strength SGNF } \uparrow \text { in } \\
\text { magnetic knee wrap group. }\end{array}$ \\
\hline $\begin{array}{l}\text { Cherian et al. } \\
(2015)[36]\end{array}$ & $\begin{array}{l}\text { Americans with knee OA ( } n \\
=5 ; \text { mean age }=69 \mathrm{y}) \\
\text { assigned to neuromuscular } \\
\text { electrical stimulation. }\end{array}$ & $\begin{array}{l}\text { Biodex dynamometer measured knee } \\
\text { flexor \& extensor strength (PT \& } \\
\text { body weight normalized PT) @ } 60^{\circ} / \mathrm{s} \text {. }\end{array}$ & Responsiveness: no difference in knee strength. \\
\hline $\begin{array}{l}\text { Cherian et al. } \\
(2015)[37]\end{array}$ & $\begin{array}{l}\text { Americans with knee OA } \\
\text { assigned to standard care ( } n \\
=10 ; \text { mean age }=54 \text { y) or } \\
\text { transcutaneous electrical } \\
\text { stimulation }(n=13 ; \text { mean } \\
\text { age }=55 \mathrm{y})\end{array}$ & $\begin{array}{l}\text { Biodex dynamometer measured knee } \\
\text { flexor \& extensor strength } \\
\text { (bodyweight adjusted PT) @ } 60^{\circ} / \mathrm{s} \text {. }\end{array}$ & $\begin{array}{l}\text { Responsiveness: SGNF } \uparrow \text { knee flexor \& extensor } \\
\text { strength in group receiving transcutaneous electrical } \\
\text { stimulation. }\end{array}$ \\
\hline $\begin{array}{l}\text { Conroy et al. } \\
(2012) \text { [38] }\end{array}$ & $\begin{array}{l}\text { Americans with knee OA }(n \\
=858 ; \text { mean age }=73.5 \mathrm{y})\end{array}$ & $\begin{array}{l}\text { Kin-Com dynamometer measured } \\
\text { concentric knee extensor strength PT) } \\
\text { @ } 60^{\circ} / \mathrm{s} \text {. }\end{array}$ & $\begin{array}{l}\text { Validity: no difference in knee extensor strength with or } \\
\text { without radiographic knee OA \& with or without pain. } \\
\text { Radiographic knee OA group knee extensor strength per } \\
\text { unit of muscle area SGNF }<\text { group without radiographic } \\
\text { knee OA. Knee extensor strength per unit of muscle area } \\
\text { for radiographic knee OA \& pain group SGNF }<\text { group } \\
\text { without radiographic knee OA or pain. }\end{array}$ \\
\hline $\begin{array}{l}\text { Cornish and } \\
\text { Peeler } \\
(2018)[39]\end{array}$ & $\begin{array}{l}\text { Canadians with knee OA } \\
\text { with creatine } \\
\text { supplementation }(n=9 ; \\
\text { mean age }=57.5 \mathrm{y}) \text { or } \\
\text { without creatine } \\
\text { supplementation }(n=8 ; \\
\text { mean age }=56.7 \mathrm{y})\end{array}$ & $\begin{array}{l}\text { Biodex System } 3 \text { measured isometric } \\
\text { knee flexor \& extensor strength (PT) } \\
@ 0,45, \& 90^{\circ} \text {. }\end{array}$ & $\begin{array}{l}\text { Responsiveness: SGNF } \downarrow \text { in isometric knee extension @ } \\
0^{\circ} \text { for both groups. No difference between groups in } \\
\text { isometric flexor or extensor isometric strength. }\end{array}$ \\
\hline $\begin{array}{l}\text { Cudejko et al. } \\
(2017)[40]\end{array}$ & $\begin{array}{l}\text { Dutch with knee OA }(n= \\
689 ; \text { mean age }=62.2 \mathrm{y})\end{array}$ & $\begin{array}{l}\text { EnKnee isokinetic dynamometer } \\
\text { measured isokinetic knee flexor \& } \\
\text { extensor strength(bodyweight } \\
\text { adjusted PT)@ } 60^{\circ} / \mathrm{s} \text {. }\end{array}$ & $\begin{array}{l}\text { Validity: SGNF association of muscle strength with } \\
\text { proprioception }(\beta=-0.48 \text { to }-0.40) \& \text { systemic } \\
\text { inflammation }(\beta=-0.42 \text { to }-0.24) \text {. }\end{array}$ \\
\hline $\begin{array}{l}\text { de Zwart et al. } \\
(2015)[41]\end{array}$ & $\begin{array}{l}\text { Dutch with knee OA }(n= \\
301 ; \text { mean age }=61.5 \mathrm{y})\end{array}$ & $\begin{array}{l}\text { EnKnee dynamometer measured knee } \\
\text { flexor \& extensor strength } \\
\text { (bodyweight adjusted PT) @ } 60^{\circ} / \mathrm{s} \text {. }\end{array}$ & $\begin{array}{l}\text { Validity: knee flexor \& extensor strength in those } \\
\text { reporting falls within previous } 3 \text { months } \mathrm{SGNF}<\text { those } \\
\text { without falls. SGNF association of knee flexor }(\mathrm{OR}= \\
0.2) \& \text { extensor }(\mathrm{OR}=0.3) \text { strength with lower falls } \\
\text { history. }\end{array}$ \\
\hline
\end{tabular}


Table 1, continued

\begin{tabular}{|c|c|c|c|}
\hline $\begin{array}{l}\text { Authorship } \\
\text { (year) }\end{array}$ & Participants & Isokinetic procedures & Findings \\
\hline $\begin{array}{l}\text { Dias et al. } \\
\text { (2017) [42] }\end{array}$ & $\begin{array}{l}\text { Brazilian females with knee } \\
\text { OA assigned to hydrotherapy } \\
(n=36 ; \text { mean age }= \\
70.8 \text { y) or control }(n=37 \\
\text { mean age }=71.0 \mathrm{y})\end{array}$ & $\begin{array}{l}\text { Biodex System } 3 \text { measured isokinetic } \\
\text { flexor \& extensor strength } \\
\text { (bodyweight adjusted PT), power, \& } \\
\text { fatigue index at } 180^{\circ} / \mathrm{s} \text {. }\end{array}$ & $\begin{array}{l}\text { Responsiveness: SGNF } \uparrow \text { in flexor \& extensor strength, } \\
\text { flexor power, \& extensor fatigue index in group receiving } \\
\text { hydrotherapy. }\end{array}$ \\
\hline $\begin{array}{l}\text { Diracoglu et } \\
\text { al. } \\
\text { (2005) [43] }\end{array}$ & $\begin{array}{l}\text { Turkish females with knee } \\
\text { OA assigned to strength } \\
\text { training with kinesthesia/ } \\
\text { balance exercises }(n=30) \\
\text { or without kinesthesia/ } \\
\text { balance exercises }(n=30)\end{array}$ & $\begin{array}{l}\text { Biodex System } 3 \text { measured knee } \\
\text { flexor \& extensor strength } \\
\text { (bodyweight adjusted PT) @ 60,180, } \\
\& 240^{\circ} / \mathrm{s} \text {. }\end{array}$ & $\begin{array}{l}\text { Responsiveness: SGNF } \uparrow \text { in isokinetic knee extensor } \\
\text { strength for both groups. Kinesthesia/balance group } \\
\text { SGNF } \uparrow \text { knee extensor strength @ 180, \& } 240^{\circ} / \mathrm{s} \text { while } \\
\text { the group without kinesthesia/balance SGNF } \uparrow \text { knee } \\
\text { extensor strength @ } 60 \& 180^{\circ} / \mathrm{s} \text {. }\end{array}$ \\
\hline $\begin{array}{l}\text { Diracoglu et } \\
\text { al. } \\
\text { (2009) [44] }\end{array}$ & $\begin{array}{l}\text { Turks with knee OA } \\
\text { assigned to hyaluronan } \\
\text { injection }(n=40 ; \text { mean age } \\
=59.4 \mathrm{y}) \text { or control placebo } \\
\text { injection }(n=20 ; \text { mean age } \\
=56.2 \mathrm{y})\end{array}$ & $\begin{array}{l}\text { Biodex System } 3 \text { Pro measured knee } \\
\text { flexor \& extensor strength (PT, \& } \\
\text { agonist/antagonist ratio) @ 60, 180, } \\
\& 240^{\circ} / \mathrm{s} \text {. }\end{array}$ & $\begin{array}{l}\text { Responsiveness: } \uparrow \text { in flexor \& extensor strength @ } 60^{\circ} / \mathrm{s} \\
\text { in the hyaluronan group SGNF }>\text { control group. No } \\
\text { between group differences in knee strength @ } 180 \text { \& } \\
240^{\circ} / \mathrm{s} \text {. }\end{array}$ \\
\hline $\begin{array}{l}\text { Diraçoglu et } \\
\text { al. } \\
\text { (2009) [45] }\end{array}$ & $\begin{array}{l}\text { Turks with bilateral knee OA } \\
(n=51 ; \text { mean age }= \\
55.6 \text { y) \& healthy controls }(n \\
=43 ; \text { mean age }=52.4 \text { y) }\end{array}$ & $\begin{array}{l}\text { Biodex System } 3 \text { Pro measured knee } \\
\text { flexor \& extensor strength } \\
\text { (bodyweight adjusted PT) @ 60,180, } \\
\& 240^{\circ} / \mathrm{s} \text {. }\end{array}$ & $\begin{array}{l}\text { Validity: knee flexor \& extensor strength in OA group @ } \\
\text { all speeds SGNF }<\text { control group. Knee flexor \& } \\
\text { extensor muscle strength for Kellgren-Lawrence stage I } \\
\text { SGNF }>\text { stage II @ all speeds. }\end{array}$ \\
\hline $\begin{array}{l}\text { Edelaar et al. } \\
\text { (2017) [46] }\end{array}$ & $\begin{array}{l}\text { Dutch with knee OA }(n= \\
562 ; \text { mean age }=62.1 \mathrm{y})\end{array}$ & $\begin{array}{l}\text { EnKnee isokinetic dynamometer } \\
\text { measured flexor \& extensor strength } \\
\text { (PT \& bodyweight adjusted PT) @ } \\
60^{\circ} / \mathrm{s} \text {. }\end{array}$ & $\begin{array}{l}\text { Validity: SGNF correlation of knee strength between } \\
\text { sides }(r=0.81) \text {. SGNF association between knee } \\
\text { strength \& Get Up and Go Test }\left(\beta=-6.45 ; r^{2}=0.32\right. \\
\text { to } 0.43) \text {. SGNF association between knee strength \& } \\
\text { Stair climb test }\left(\beta=-7.53 ; r^{2}=0.31 \text { to } 0.45\right) \text {. SGNF } \\
\text { association between knee strength \& Stair descent test } \\
\left(\beta=-9.02 ; r^{2}=0.30 \text { to } 0.39\right) \text {. }\end{array}$ \\
\hline $\begin{array}{l}\text { Eyigör et al. } \\
\text { (2004) [47] }\end{array}$ & $\begin{array}{l}\text { Turks with bilateral knee OA } \\
\text { assigned to isokinetic } \\
\text { exercise }(n=21 ; \text { mean age } \\
=53.1 \text { y) or progressive } \\
\text { resistance exercise }(n=18 ; \\
\text { mean age }=51.9 \text { y) }\end{array}$ & $\begin{array}{l}\text { Cybex Norm Dynamometer measured } \\
\text { knee flexor \& extensor strength @ } 60 \text {, } \\
90,120, \& 180^{\circ} / \mathrm{s} \text {. }\end{array}$ & $\begin{array}{l}\text { Responsiveness: SGNF } \uparrow \text { isokinetic flexor \& extensor } \\
\text { strength in both groups without between group } \\
\text { differences. }\end{array}$ \\
\hline $\begin{array}{l}\text { Germanou et } \\
\text { al. } \\
\text { (2013) [48] }\end{array}$ & $\begin{array}{l}\text { Greeks with knee } \mathrm{OA}(n= \\
10 ; \text { mean age }=58.9 \mathrm{y}) \& \\
\text { age-weight matched controls } \\
(n=10 ; \text { mean age }= \\
62.4 \text { y) assigned to isokinetic } \\
\text { exercise. }\end{array}$ & $\begin{array}{l}\text { Cybex } 6000 \text { dynamometer measured } \\
\text { concentric flexor \& extensor (PT) @ } \\
90,120, \& 150^{\circ} / \mathrm{s} .\end{array}$ & $\begin{array}{l}\text { Validity: knee flexor \& extensor strength in knee OA } \\
\text { group SGNF < control group. }\end{array}$ \\
\hline $\begin{array}{l}\text { Gkrilias et al. } \\
\text { (2018) [49] }\end{array}$ & $\begin{array}{l}\text { Greeks with knee OA }(n= \\
40 ; \text { mean age }=61.7 \mathrm{y})\end{array}$ & $\begin{array}{l}\text { Biodex System } 3 \text { measured isokinetic } \\
\text { flexor \& extensor strength } \\
\text { (bodyweight adjusted PT) @ } 120 \& \\
180^{\circ} / \mathrm{s} \text {. }\end{array}$ & $\begin{array}{l}\text { Validity: SGNF correlation between isokinetic flexor \& } \\
\text { extensor strength @ both speeds with } 6 \text { minute Walk Test } \\
(r=0.618 \text { to } 0.666) \text {, Timed Up and Go Test }(r= \\
-0.625 \text { to }-0.530), 30 \text { second Chair Stand Test }(r= \\
0.509 \text { to } 0.557), \& 12 \text { Stair Climb Test }(r=-0.671 \text { to } \\
-0.624) \text {. Functional measures predicted isokinetic } \\
\text { strength }\left(r^{2}=0.497 \text { to } 0.607\right) \text {. }\end{array}$ \\
\hline $\begin{array}{l}\text { Glass et al. } \\
\text { (2013) [50] }\end{array}$ & $\begin{array}{l}\text { Americans with knee OA or } \\
\text { risk factors for knee OA ( } n \\
=2404 ; \text { mean age }= \\
62.2 \mathrm{y})\end{array}$ & $\begin{array}{l}\text { Cybex } 350 \text { dynamometer measured } \\
\text { knee flexor \& extensor strength (PT) } \\
\text { @ } 60^{\circ} / \mathrm{s} \text {. }\end{array}$ & $\begin{array}{l}\text { Validity: knee strength in males SGNF }>\text { females. } \\
\text { Responsiveness: lower knee extensor strength SGNF }> \\
\text { risk }(\mathrm{RR}=1.23 \text { to } 1.28) \text { of worsening knee pain in } \\
\text { females but not males. }\end{array}$ \\
\hline $\begin{array}{l}\text { Gökçen et al. } \\
\text { (2016) [51] }\end{array}$ & $\begin{array}{l}\text { Turks with knee OA }(n= \\
152 ; \text { mean age }=57.3 \mathrm{y})\end{array}$ & $\begin{array}{l}\text { NORM } 6000 \text { dynamometer measured } \\
\text { knee flexor \& extensor strength (PT) } \\
\text { @ @ 60\% } / \mathrm{s} \& \text { isometric strength (PT) } \\
60^{\circ} .\end{array}$ & $\begin{array}{l}\text { Validity: Female knee strength SGNF }<\text { males. SGNF } \\
\text { inverse correlation of knee strength with pain }(r= \\
-0.33 \text { to }-0.22) \text {, comorbidity }(r=-0.32 \text { to }-0.22) \& \\
\text { WOMAC score }(r=-0.37 \text { to }-0.21) \text {. No difference in } \\
\text { knee strength by Kellgren-Lawrence grade. }\end{array}$ \\
\hline
\end{tabular}


Table 1, continued

\begin{tabular}{|c|c|c|c|}
\hline $\begin{array}{c}\text { Authorship } \\
\text { (year) }\end{array}$ & Participants & Isokinetic procedures & Findings \\
\hline $\begin{array}{l}\text { Gur et al. } \\
(2002) \text { [52] }\end{array}$ & $\begin{array}{l}\text { Turks with knee OA } \\
\text { assigned to concentric }(n= \\
9 ; \text { mean age }=56 \mathrm{y}) \\
\text { concentric-eccentric }(n=8 ; \\
\text { mean age }=55 \mathrm{y}), \text { or no } \\
\text { exercise control }(n=6 ; \\
\text { mean age }=57 \mathrm{y})\end{array}$ & $\begin{array}{l}\text { Cybex } 6000 \text { dynamometer measured } \\
\text { concentric \& eccentric flexor \& } \\
\text { extensor strength (PT) @ 60,120, \& } \\
180^{\circ} / \mathrm{s} .\end{array}$ & $\begin{array}{l}\text { Responsiveness: SGNF } \uparrow \text { concentric extensor strength @ } \\
120, \& 180^{\circ} / \mathrm{s} \text { in concentric group only. SGNF } \uparrow \\
\text { eccentric strength in concentric-eccentric group at all } \\
\text { speeds, \& concentric group @ } 120^{\circ} / \mathrm{s} \text {. SGNF } \uparrow \\
\text { concentric flexor strength for both exercise groups. } \\
\text { SGNF } \uparrow \text { in eccentric flexor strength @ } 60 \& 120^{\circ} / \mathrm{s} \text { for } \\
\text { both exercise groups but only the concentric-eccentric } \\
\text { group @ } 180^{\circ} / \mathrm{s} \text {. }\end{array}$ \\
\hline $\begin{array}{l}\text { Gur and } \\
\text { Cakin } \\
(2003)[53]\end{array}$ & $\begin{array}{l}\text { Turkish females with } \\
\text { bilateral knee OA }(n=18 \\
\text { mean age }=56 \text { y) }\end{array}$ & $\begin{array}{l}\text { Cybex } 6000 \text { dynamometer measured } \\
\text { concentric \& eccentric flexor \& } \\
\text { extensor strength (PT) @ 60,120, \& } \\
180^{\circ} / \mathrm{s} .\end{array}$ & $\begin{array}{l}\text { Validity: eccentric strength for knee flexors \& extensors } \\
\text { SGNF }>\text { concentric @ all speeds. SGNF correlation of } \\
\text { eccentric to concentric strength for flexors }(r=0.36 \text { to } \\
0.87) \& \text { extensors ( } r=0.73 \text { to } 0.86) @ \text { all speeds. SGNF } \\
\text { correlation between concentric flexor \& extensor strength } \\
\text { ( } r=0.63 \text { to } 0.83) \& \text { eccentric flexor \& extensor strength } \\
\text { ( } r=0.61 \text { to } 0.88) \text {. SGNF correlation between quadriceps } \\
\text { cross sectional area with concentric \& eccentric exten- } \\
\text { sor strength ( } r=0.68 \text { to } 0.78) \& \text { hamstrings cross sec- } \\
\text { tional area with concentric } \& \text { eccentric flexor strength } \\
(r=0.49 \text { to } 0.73) \text {. Variation in strength was partially } \\
\text { explained by cross sectional area of flexors }\left(r^{2}=0.46\right. \\
\text { to } 0.61) \& \text { extensors }\left(r^{2}=0.24 \text { to } 0.54\right) \text { respectively. } \\
\text { SGNF correlation of stair climbing time with eccentric } \\
\text { flexor to concentric extensor ratio }(r=0.71 \text { to } 0.73) \& \\
\text { stair descent time with concentric flexor to eccentric ex- } \\
\text { tensor ratio }(r=0.68 \text { to } 0.77) \text {. Stair climbing time was } \\
\text { predicted by eccentric flexor to concentric extensor ration } \\
\left(r^{2}=0.81\right) \text {, eccentric flexor strength }\left(r^{2}=0.48\right) \& \text { con- } \\
\text { centric flexor to concentric extensor ratio }\left(r^{2}=0.45\right) \text {. } \\
\text { Stair descent time was predicted by concentric flexor to } \\
\text { eccentric extensor ratio }\left(r^{2}=0.61\right) \& \text { concentric flexor } \\
\text { to concentric extensor ratio }\left(r^{2}=0.45\right) \text {. } \\
\text { Reliability: test-retest }(r=0.72 \text { to } 0.95) \text { for all strength } \\
\text { measures. }\end{array}$ \\
\hline $\begin{array}{l}\text { Ha et al. } \\
(2018)[54]\end{array}$ & $\begin{array}{l}\text { Korean females with knee } \\
\text { OA assigned to aquatic } \\
\text { therapy ( } n=9 ; \text { mean age }= \\
60.9 \text { y) or non-exercise } \\
\text { control ( } n=8 ; \text { mean age }= \\
61.3 \text { y). }\end{array}$ & $\begin{array}{l}\text { Biodex System } 3 \text { measured isokinetic } \\
\text { knee flexor \& extensor strength } \\
\text { (bodyweight adjusted PT) @ } 60^{\circ} / \mathrm{s} \text {. }\end{array}$ & $\begin{array}{l}\text { Responsiveness: SGNF } \uparrow \text { knee extensor strength in } \\
\text { aquatic therapy group, although no difference between } \\
\text { groups. }\end{array}$ \\
\hline $\begin{array}{l}\text { Hall et al. } \\
(2018) \text { [55] }\end{array}$ & $\begin{array}{l}\text { Australians with knee OA } \\
\text { assigned to exercise }(n= \\
49 ; \text { mean age }=65.7 \mathrm{y}) \text { or } \\
\text { control }(n=48 ; \text { mean age } \\
=63.8 \mathrm{y})\end{array}$ & $\begin{array}{l}\text { KinCom } 125 \text {-AP measured isometric } \\
\text { knee extensor strength (bodyweight } \\
\text { adjusted PT) @ } 60^{\circ} \text {. }\end{array}$ & $\begin{array}{l}\text { Responsiveness: knee extensor strength in exercise } \\
\text { group SGNF }>\text { control group. } \uparrow \text { in knee extensor } \\
\text { strength accounted for } 38 \% \& 60 \% \text { of variability in } \\
\text { WOMAC pain \& function. }\end{array}$ \\
\hline $\begin{array}{l}\text { Harper } \\
(2019)[56]\end{array}$ & $\begin{array}{l}\text { Americans with knee OA } \\
\text { assigned to moderate } \\
\text { intensity ( } n=19 \text {; mean age } \\
=69.1 \mathrm{y}) \text { or low load blood } \\
\text { flow restriction resistance } \\
\text { training }(n=16 \text {; mean age } \\
=67.2 \mathrm{y}) .\end{array}$ & $\begin{array}{l}\text { Biodex system measured knee } \\
\text { extensor isokinetic strength (PT) @ } \\
60^{\circ}, 90^{\circ}, \& 120^{\circ} / \mathrm{s} \text {. }\end{array}$ & $\begin{array}{l}\text { Responsiveness: SGNF } \uparrow \text { knee extensor strength @ all } \\
\text { three speeds for both groups, but no between group } \\
\text { differences. }\end{array}$ \\
\hline $\begin{array}{l}\text { Huang et al. } \\
(2003) \text { [57] }\end{array}$ & $\begin{array}{l}\text { Taiwanese with bilateral } \\
\text { knee OA (mean age }=62 \text { y) } \\
\text { assigned to isokinetic } \\
\text { exercise }(n=33) \text {, isotonic } \\
\text { exercise }(n=33) \text {, isometric } \\
\text { exercise }(n=33) \text {, or } \\
\text { non-exercise control }(n= \\
\text { 33). }\end{array}$ & $\begin{array}{l}\text { Kin-Com } 505 \text { dynamometer } \\
\text { measured eccentric \& concentric } \\
\text { flexor \& extensor strength (PT) @ } 60 \\
\& 180^{\circ} / \mathrm{s} \text {. }\end{array}$ & $\begin{array}{l}\text { Responsiveness: SGNF } \uparrow \text { in strength @ } 60^{\circ} / \mathrm{s} \text { in } \\
\text { isokinetic \& isotonic groups @ } 8 \text { weeks \& } 1 \text { year, \& only } \\
1 \text { year for isometric group. SGNF } \uparrow \text { in strength for } \\
\text { isokinetic group only @ } 180^{\circ} / \mathrm{s} @ 8 \text { weeks \& } 1 \text { year. }\end{array}$ \\
\hline
\end{tabular}


Table 1 , continued

\begin{tabular}{|c|c|c|c|}
\hline $\begin{array}{l}\text { Authorship } \\
\text { (year) }\end{array}$ & Participants & Isokinetic procedures & Findings \\
\hline $\begin{array}{l}\text { Huang et al. } \\
\text { (2005) [58] }\end{array}$ & $\begin{array}{l}\text { Taiwanese with bilateral } \\
\text { knee OA (mean age }= \\
65.0 \text { y) assigned to isokinetic } \\
\text { exercise }(n=35) \text {, isokinetic } \\
\text { exercise \& pulse ultrasound } \\
(n=35), \text { isokinetic exercise } \\
\& \text { pulse ultrasound \& } \\
\text { hyaluronan injection }(n= \\
35), \text { or non-exercise control } \\
(n=35) .\end{array}$ & $\begin{array}{l}\text { Kin-Com } 505 \text { dynamometer } \\
\text { measured concentric \& eccentric } \\
\text { flexor \& extensor strength (PT) @ } 60 \\
\& 180^{\circ} / \mathrm{s} \text {. }\end{array}$ & $\begin{array}{l}\text { Responsiveness: SGNF } \uparrow \text { in concentric \& eccentric } \\
\text { flexor \& extensor strength @ } 60 \& 180^{\circ} / \mathrm{s} \text { in all exercise } \\
\text { groups @ } 8 \text { weeks \& } 1 \text { year, with group receiving } \\
\text { exercise, pulse ultrasound \& hyaluronan SGNF }>\text { other } \\
\text { groups. }\end{array}$ \\
\hline $\begin{array}{l}\text { Huang et al. } \\
\text { (2005) [59] }\end{array}$ & $\begin{array}{l}\text { Taiwanese with knee OA ( } n \\
=120 ; \text { mean age }=62.0 \mathrm{y}) \\
\text { assigned to control group, } \\
\text { isokinetic exercise, or } \\
\text { isokinetic exercise with } \\
\text { continuous, or isokinetic } \\
\text { exercise with pulsed } \\
\text { ultrasound. }\end{array}$ & $\begin{array}{l}\text { Kin-Com } 505 \text { dynamometer } \\
\text { measured concentric \& eccentric knee } \\
\text { flexion \& extension strength (PT) @ } \\
60 \& 180^{\circ} / \mathrm{s} \text {. }\end{array}$ & $\begin{array}{l}\text { Responsiveness: SGNF } \uparrow \text { flexor \& extensor strength @ } \\
60^{\circ} / \mathrm{s} \text { after treatment \& } @ \text { follow-up for groups receiving } \\
\text { isokinetic exercise \& either pulsed or continuous } \\
\text { ultrasound compared to other groups. SGNF } \uparrow \text { flexor \& } \\
\text { extensor strength @ } 60^{\circ} / \mathrm{s} @ \text { follow-up in isokinetic } \\
\text { only compared to control group. } \uparrow \text { knee flexor \& } \\
\text { extensor strength @ } 180^{\circ} / \mathrm{s} \text { in group receiving isokinetic } \\
\text { exercise \& pulsed ultrasound SGNF }>\text { other groups. }\end{array}$ \\
\hline $\begin{array}{l}\text { Hurley and } \\
\text { Newham } \\
\text { (1993) [60] }\end{array}$ & $\begin{array}{l}\text { British with knee OA ( } n= \\
10 ; \text { mean age }=56 \mathrm{y}) \\
\text { assigned to exercise } \\
\text { including isokinetic training. }\end{array}$ & $\begin{array}{l}\text { Cybex System II + dynamometer } \\
\text { measured extensor strength (PT) @ } \\
30,60,90,120 \& 180^{\circ} / \mathrm{s} \& \text { isometric } \\
\text { extensor strength }(\mathrm{PT}) @ 90^{\circ} .\end{array}$ & $\begin{array}{l}\text { Validity: isometric knee extensor strength in knee OA } \\
\text { SGNF < age predicted norms \& contralateral knee with- } \\
\text { out OA. } \\
\text { Responsiveness: SGNF } \uparrow \text { knee extensor strength @ } 30^{\circ} / \mathrm{s} \\
\text { in knees without OA. SGNF improvement in knee ex- } \\
\text { tensor strength deficits in knee with OA compared to } \\
\text { contralateral knee @ } 30,60,90, \& 120^{\circ} / \mathrm{s} \text {. } \\
\text { SGNF } \uparrow \text { in isometric knee extensor strength to achieve } \\
\text { predicted normative values. } \\
\text { SGNF }<\text { knee extensor strength compared to } \\
\text { contralateral knee without OA @ all speeds remained } \\
\text { after training. No difference in isometric knee extensor } \\
\text { strength between knee OA \& contralateral knee. }\end{array}$ \\
\hline $\begin{array}{l}\text { Jadelis et al. } \\
\text { (2001) [61] }\end{array}$ & $\begin{array}{l}\text { Americans with knee OA }(n \\
=480 ; \text { mean age }=71.8 \mathrm{y})\end{array}$ & $\begin{array}{l}\text { Kin-Com } 125 \mathrm{E} \text { dynamometer } \\
\text { measured concentric \& eccentric knee } \\
\text { flexor \& extensor strength (PT) @ } \\
30 \% \text { velocity. }\end{array}$ & $\begin{array}{l}\text { Validity: correlation of knee flexor \& extensor strength } \\
(r=0.97), \& \text { knee strength with ankle strength }(r= \\
0.54 \text { to } 0.68) \text {. Knee strength in males SGNF }>\text { females. } \\
\text { Knee strength partially explained the variance in } \\
\text { anterioposterior balance }\left(r^{2}=18.4 \%\right) \text {. }\end{array}$ \\
\hline $\begin{array}{l}\text { Kean et al. } \\
(2010)[62]\end{array}$ & $\begin{array}{l}\text { Canadians with knee OA }(n \\
=20 ; \text { mean age }=53.6 \mathrm{y})\end{array}$ & $\begin{array}{l}\text { Biodex System } 3 \text { dynamometer } \\
\text { measured concentric knee extensor } \\
\text { strength (bodyweight adjusted PT \& } \\
\text { body size adjusted PT) @ 60\% } / \text { s } \\
\text { isometric extensor strength } \\
\text { (bodyweight adjusted PT \& body size } \\
\text { adjusted PT)@ } 90^{\circ} .\end{array}$ & $\begin{array}{l}\text { Reliability: test-retest isokinetic knee extensor strength } \\
(\mathrm{ICC}=0.93 ; \mathrm{MDC}=33.90 \mathrm{Nm}) \& \text { isometric knee } \\
\text { extensor strength }(\mathrm{ICC}=0.98 ; \mathrm{MDC}=25.02 \mathrm{Nm})\end{array}$ \\
\hline $\begin{array}{l}\text { Kim et al. } \\
(2018)[63]\end{array}$ & $\begin{array}{l}\text { Taiwanese females with } \\
\text { bilateral knee OA \& no } \\
\text { swelling }(n=40 ; \text { mean age } \\
=68.3 \mathrm{y})\end{array}$ & $\begin{array}{l}\text { CSMI isokinetic dynamometer } \\
\text { measured maximal knee flexion \& } \\
\text { extension strength (PT) @ } 60 \text { \& } \\
180^{\circ} / \mathrm{s} \text {. }\end{array}$ & $\begin{array}{l}\text { Validity: knee extensor strength in painful knees SGNF } \\
\text { < non-painful knees. SGNF inverse correlation for knee } \\
\text { extensor strength with dynamic balance stability }(r= \\
-0.58 \text { to }-0.44) \text {. No correlation between knee strength } \\
\text { \& proprioception. }\end{array}$ \\
\hline $\begin{array}{l}\text { King et al. } \\
\text { (2008) [64] }\end{array}$ & $\begin{array}{l}\text { Canadians with knee } \mathrm{OA} \& \\
\text { varus malalignment }(n=14 \text {; } \\
\text { mean age }=48.4 \mathrm{y}) \text { assigned } \\
\text { to isokinetic resistance } \\
\text { training. }\end{array}$ & $\begin{array}{l}\text { Biodex System } 3 \text { measured concentric } \\
\text { knee flexor \& extensor strength (PT } \\
\& \text { work)@60,90,120, \& } 180^{\circ} / \mathrm{s} \text {. }\end{array}$ & $\begin{array}{l}\text { Validity: knee strength in affected limb SGNF }<\text { unaf- } \\
\text { fected at all time points. } \\
\text { Responsiveness: SGNF } \uparrow \text { in knee flexor \& extensor } \\
\text { strength at all speeds at week } 3 \text {, no further } \uparrow \text { afterward. }\end{array}$ \\
\hline $\begin{array}{l}\text { Koeckhoven } \\
\text { et al. } \\
\text { (2016) [65] }\end{array}$ & $\begin{array}{l}\text { Dutch with knee OA }(n= \\
319 ; \text { mean age }=60.5 \mathrm{y})\end{array}$ & $\begin{array}{l}\text { En-Knee dynamometer measured } \\
\text { knee flexor \& extensor strength (body } \\
\text { weight adjusted PT) @ } 60^{\circ} / \mathrm{s} \text {. }\end{array}$ & $\begin{array}{l}\text { Validity: SGNF correlation between right \& left limb } \\
\text { strength }(r>0.75) \text {. Individuals with serum } 25 \text {-hydroxy } \\
\text { vitamin D levels }<50 \text { nmol/1 had SGNF } \downarrow \text { strength. } \\
\text { SGNF association for strength with } 25 \text {-hydroxy vitamin } \\
\text { D levels }(\beta=0.03), \& \text { BMI }(\beta=-0.448) \text {. }\end{array}$ \\
\hline
\end{tabular}


Table 1, continued

\begin{tabular}{|c|c|c|c|}
\hline $\begin{array}{l}\text { Authorship } \\
\text { (year) }\end{array}$ & Participants & Isokinetic procedures & Findings \\
\hline $\begin{array}{l}\text { Kumar et al. } \\
(2014)[66]\end{array}$ & $\begin{array}{l}\text { Americans with radiographic } \\
\text { knee OA }(n=30 ; \text { mean age } \\
=57.7 \mathrm{y}) \text { or without } \\
\text { radiographic knee OA }(n= \\
66 ; \text { mean age }=50.7 \mathrm{y})\end{array}$ & $\begin{array}{l}\text { Primus RS dynamometer measured } \\
\text { knee flexor \& extensor strength } \\
\text { (bodyweight adjusted PT) @ } 120^{\circ} / \mathrm{s} \text {, } \\
\text { \& isometric knee flexor \& extensor } \\
\text { strength (bodyweight adjusted PT) @ } \\
70^{\circ} \text {. }\end{array}$ & $\begin{array}{l}\text { Validity: knee extensor strength in knee OA group SGNF } \\
<\text { control group. No difference between groups for knee } \\
\text { flexor strength. SGNF inverse correlation between } \\
\text { isometric knee extensor strength \& age }(r=-0.36) \text {, } \\
\text { Kellgren-Lawrence grade }(r=-0.23), \& \text { total cartilage } \\
(r=-0.16) \text {. }\end{array}$ \\
\hline $\begin{array}{l}\text { Lankhorst et } \\
\text { al. } \\
\text { (1985) [67] }\end{array}$ & $\begin{array}{l}\text { Dutch with knee OA }(n= \\
39 ; \text { mean age }=62.4 \text { y) }\end{array}$ & $\begin{array}{l}\text { Cybex System II dynamometer } \\
\text { measured knee flexor \& extensor } \\
\text { strength (PT) @ 30,60,120, \& } \\
180^{\circ} / \mathrm{s} \& \text { isometric knee extension } \\
\text { strength (PT) @ } 90^{\circ} .\end{array}$ & $\begin{array}{l}\text { Validity: knee strength on affected knee SGNF }< \\
\text { unaffected contralateral knee }(65-83 \%) \text {. SGNF } \\
\text { correlation between isometric \& isokinetic strength @ all } \\
\text { speeds }(r=0.80 \text { to } 0.95) \text {. SGNF inverse correlation of } \\
\text { walking time with knee extensor strength @ } 30^{\circ} / \mathrm{s}(r= \\
-0.46) \& \text { isometric knee extensor strength }(r=-0.41) \text {. } \\
\text { Variability in walking time, functional capacity score, } \\
\text { stair climb test, \& mean pain score were partially } \\
\text { explained by a combination of knee extensor strength \& } \\
\text { pain }\left(r^{2}=0.23 \text { to } 0.38\right) \text {. }\end{array}$ \\
\hline $\begin{array}{l}\text { Lee et al. } \\
(2015)[68]\end{array}$ & $\begin{array}{l}\text { Koreans with knee OA }(n= \\
35 ; \text { mean age }=57.8 \text { y) }\end{array}$ & $\begin{array}{l}\text { Biodex System } 4 \text { dynamometer } \\
\text { measured knee flexor \& extensor } \\
\text { strength (bodyweight adjusted PT \& } \\
\text { work) @ } 60 \& 180^{\circ} / \mathrm{s} \text {. }\end{array}$ & $\begin{array}{l}\text { Validity: SGNF correlation of knee flexor }(r=0.43) \& \\
\text { extensor }(r=0.43) \text { work @ } 180^{\circ} / \mathrm{s} \text { with adduction } \\
\text { moment. No correlation between knee flexor or extensor } \\
\text { PT with adduction moment. }\end{array}$ \\
\hline $\begin{array}{l}\text { Levinger et } \\
\text { al. } \\
(2018)[69]\end{array}$ & $\begin{array}{l}\text { Australians with knee OA }(n \\
=24 ; \text { mean age }=68.6 \mathrm{y})\end{array}$ & $\begin{array}{l}\text { Biodex System } 2 \text { measured isokinetic } \\
\text { knee extensor strength (bodyweight \& } \\
\text { height adjusted PT) @ } 90 \& 180^{\circ} / \mathrm{s}, \\
\& \text { flexor strength (bodyweight \& } \\
\text { height adjusted PT) @ } 120^{\circ} / \mathrm{s} .\end{array}$ & $\begin{array}{l}\text { Validity: individuals with multiple stepping strategy after } \\
\text { induced forward fall had SGNF } \downarrow \text { isokinetic knee } \\
\text { extensor strength @ } 180^{\circ} / \mathrm{s} \text { than those with fewer steps. } \\
\text { No difference in other strength measures by stepping } \\
\text { strategy. }\end{array}$ \\
\hline $\begin{array}{l}\text { Lim et al. } \\
(2010)[70]\end{array}$ & $\begin{array}{l}\text { Obese Koreans with knee } \\
\text { OA assigned to aquatic } \\
\text { exercise }(n=26 ; \text { mean age } \\
=65.7 \mathrm{y}), \text { land-based } \\
\text { exercise }(n=25 ; \text { mean age } \\
=67.7 \mathrm{y}), \text { or control ( } n= \\
24 ; \text { mean age }=63.3 \mathrm{y} .\end{array}$ & $\begin{array}{l}\text { Biodex dynamometer measured knee } \\
\text { flexor \& extensor strength (PT) @ } \\
60^{\circ} / \mathrm{s} \text {. }\end{array}$ & $\begin{array}{l}\text { Responsiveness: No change in knee strength for any } \\
\text { group. }\end{array}$ \\
\hline $\begin{array}{l}\text { Lim et al. } \\
(2015)[71]\end{array}$ & $\begin{array}{l}\text { Koreans with knee OA }(n= \\
40 ; \text { mean age }=56.7 \mathrm{y})\end{array}$ & $\begin{array}{l}\text { Primus RS dynamometer measured } \\
\text { isometric extensor strength } \\
\text { (bodyweight adjusted PT) @ } 60^{\circ} .\end{array}$ & $\begin{array}{l}\text { Validity: no difference in isometric knee extension } \\
\text { strength by radiographic knee alignment. }\end{array}$ \\
\hline $\begin{array}{l}\text { Madsen et al. } \\
\text { (1996) [72] }\end{array}$ & $\begin{array}{l}\text { Danish with knee OA } \\
\text { awaiting knee arthroplasty } \\
(n=23 ; \text { median age }= \\
69 \mathrm{y}) .\end{array}$ & $\begin{array}{l}\text { Cybex } 6000 \text { dynamometer measured } \\
\text { knee flexor \& extensor strength (PT) } \\
\text { @ } 30 \& 120^{\circ} / \text { s \& isometric knee } \\
\text { flexor \& extensor strength (PT) @ } \\
75^{\circ} \text {. }\end{array}$ & $\begin{array}{l}\text { Validity: knee extensor strength SGNF }>\text { flexor strength. } \\
\text { Knee extensor strength @ } 120^{\circ} / \mathrm{s} \text { SGNF }<30^{\circ} / \mathrm{s} \text { or iso- } \\
\text { metric strength. } \\
\text { Reliability: test-retest knee flexor strength @ } 120^{\circ} / \mathrm{s} \\
(\text { ICC }=0.81 \text { to } 0.92) .\end{array}$ \\
\hline $\begin{array}{l}\text { Malas et al. } \\
(2013)[73]\end{array}$ & $\begin{array}{l}\text { Turks with knee OA } \\
\text { assigned to isokinetic ( } n= \\
20 ; \text { mean age }=56.2 \mathrm{y}), \\
\text { isometric }(n=22 ; \text { mean age } \\
=61.2 \mathrm{y}), \text { or isotonic } \\
\text { exercise }(n=19 ; \text { mean age } \\
=59.1 \mathrm{y})\end{array}$ & $\begin{array}{l}\text { Biodex System } 3 \text { dynamometer } \\
\text { measured concentric flexor \& } \\
\text { extensor strength (body weight } \\
\text { adjusted PT) @ } 60^{\circ} / \mathrm{s} \text {. }\end{array}$ & $\begin{array}{l}\text { Responsiveness: SGNF } \uparrow \text { knee extensor strength in } \\
\text { trained \& contralateral knee for isometric group only. No } \\
\text { within group differences for knee flexor strength. No } \\
\text { difference in knee strength between groups after training. }\end{array}$ \\
\hline $\begin{array}{l}\text { Maly et al. } \\
(2006)[74]\end{array}$ & $\begin{array}{l}\text { Canadians with knee OA }(n \\
=54 ; \text { mean age }=68.3 \mathrm{y})\end{array}$ & $\begin{array}{l}\text { Biodex System } 3 \text { measured concentric } \\
\text { flexor \& extensor strength (PT) @ } \\
60^{\circ} / \mathrm{s} \text {. }\end{array}$ & $\begin{array}{l}\text { Validity: SGNF correlation of Functional Self Efficacy } \\
\text { scores with knee extensor strength }(r=0.35) \& \text { flexor } \\
\text { strength }(r=0.39) \text {. SGNF correlation of flexor with } \\
\text { extensor strength }(r=0.79) \text {. Variance in Functional Self } \\
\text { Efficacy scores is partially explained by stiffness, flexor } \\
\text { strength, age, \& depression }\left(r^{2}=0.51\right) \text {. }\end{array}$ \\
\hline $\begin{array}{l}\text { Maly et al. } \\
(2008)[75] \\
\text { Matsuno et al. } \\
(1997)[76]\end{array}$ & $\begin{array}{l}\text { Canadians with knee OA }(n \\
=53 ; \text { mean age }=68.5 \mathrm{y}) \\
\text { Japanese with knee OA }(n= \\
20 ; \text { mean age }=76.6 \mathrm{y}) \\
\text { assigned to knee bracing. }\end{array}$ & $\begin{array}{l}\text { Biodex System } 3 \text { measured knee } \\
\text { extensor strength (PT) @ } 60^{\circ} / \mathrm{s} \text {. } \\
\text { KIN-500-HX dynamometer measured } \\
\text { knee extensor strength (PT) @ 30\% } / \mathrm{s} \text {. }\end{array}$ & $\begin{array}{l}\text { Validity: muscle strength did not contribute to } \\
\text { explanation of pain variability. } \\
\text { Responsiveness: SGNF } \uparrow \text { in knee extensor strength with } \\
\text { knee brace. }\end{array}$ \\
\hline
\end{tabular}


Table 1, continued

\begin{tabular}{|c|c|c|c|}
\hline $\begin{array}{l}\text { Authorship } \\
\text { (year) }\end{array}$ & Participants & Isokinetic procedures & Findings \\
\hline $\begin{array}{l}\text { Matsuse et al. } \\
\text { (2017) [77] }\end{array}$ & $\begin{array}{l}\text { Japanese with knee OA }(n= \\
11 ; \text { mean age }=74.0 \mathrm{y}) \\
\text { assigned to electrical } \\
\text { stimulation with walking. }\end{array}$ & $\begin{array}{l}\text { Biodex System } 3 \text { measured isokinetic } \\
\text { knee flexor \& extensor strength } \\
\text { (bodyweight adjusted PT) @ } 60^{\circ} / \mathrm{s} \text {. }\end{array}$ & Responsiveness: SGNF $\uparrow$ extensor \& flexor strength. \\
\hline $\begin{array}{l}\text { Maurer et al. } \\
(1999)[78]\end{array}$ & $\begin{array}{l}\text { Americans with knee OA } \\
\text { assigned to an educational } \\
\text { intervention }(n=49 ; \text { mean } \\
\text { age }=64.5 \mathrm{y}) \text { or isokinetic } \\
\text { exercise }(n=49 ; \text { mean age } \\
=66.3 \mathrm{y} .\end{array}$ & $\begin{array}{l}\text { Biodex dynamometer measured knee } \\
\text { extensor strength (PT) @ } 90 \text { \& } \\
120^{\circ} / \mathrm{s}, \text { \& isometric knee extensor } \\
\text { strength (PT) @ } 90^{\circ} .\end{array}$ & $\begin{array}{l}\text { Responsiveness: SGNF } \uparrow \text { knee extensor isometric } \\
\text { strength \& isokinetic strength @ } 90^{\circ} / \mathrm{s} \text { in both the } \\
\text { exercise \& education groups, with only the exercise } \\
\text { group } \uparrow @ 120^{\circ} / \mathrm{s} \text {. SGNF association of } \uparrow \text { knee extensor } \\
\text { strength @ } 120^{\circ} / \mathrm{s} \text { with } \downarrow \text { WOMAC pain, function, \& } \\
\text { total score. }\end{array}$ \\
\hline $\begin{array}{l}\text { Messier et al. } \\
(1992) \text { [79] }\end{array}$ & $\begin{array}{l}\text { Americans with knee OA }(n \\
=15 ; \text { mean age }=58.7 \mathrm{y}) \text { or } \\
\text { without knee OA }(n=15 ; \\
\text { mean age }=58.1 \mathrm{y}) .\end{array}$ & $\begin{array}{l}\text { Cybex II }+ \text { dynamometer measured } \\
\text { knee flexor \& extensor strength } \\
\text { (bodyweight adjusted PT \& peak } \\
\text { flexor-extensor ratio) @ } 60^{\circ} / \mathrm{s} .\end{array}$ & $\begin{array}{l}\text { Validity: knee flexor \& extensor strength in OA group } \\
\text { SGNF }<\text { control group. Non-dominant leg flexor \& } \\
\text { extensor strength SGNF }<\text { dominant leg in the OA } \\
\text { group. }\end{array}$ \\
\hline $\begin{array}{l}\text { Messier et al. } \\
(2000)[80]\end{array}$ & $\begin{array}{l}\text { Obese Americans with knee } \\
\text { OA assigned to exercise \& }\end{array}$ & $\begin{array}{l}\text { Kin-Com } 125 \mathrm{E} \text { isokinetic } \\
\text { dynamometer measured flexor \& }\end{array}$ & $\begin{array}{l}\text { Responsiveness: SGNF } \uparrow \text { in flexor \& extensor strength } \\
\text { in both groups without between group differences. }\end{array}$ \\
\hline
\end{tabular}
diet $(n=13$; mean age $=\quad$ extensor strength (mean torque) @ $69 \mathrm{y})$ or exercise alone $(n=30 \% \mathrm{~s}$ 11 ; mean age $=67 \mathrm{y}$ ).

Messier et al. Older Americans with knee Kin-Com 125E dynamometer (2002) [81] OA $(n=480$; mean age measured concentric \& eccentric knee $71.8 \mathrm{y})$. flexor \& extensor strength (PT) @ $30 \%$ s.

Validity: SGNF association between knee \& ankle strength @ baseline.

Responsiveness: SGNF $\downarrow$ in knee flexor \& extensor strength. SGNF association between knee \& ankle strength @ 30 months. Greater knee strength at baseline was SGNF associated with less decline in balance @ 30 months.

Mikesky et al. Older Americans assigned to Kin-Com III dynamometer measured Validity: knee extensor strength in males without knee (2006) [82] strength training $(n=113$; concentric flexor \& extensor strength mean age $=69.4 \mathrm{y})$ or range $(\mathrm{PT}) @ 60 \& 120^{\circ} / \mathrm{s}$. of motion $(n=108$; mean age $=68.6 \mathrm{y})$.

$\overline{\mathrm{OA}} \mathrm{SGNF}>$ males with knee OA, no difference in knee flexor strength. Knee flexor \& extensor strength in females without pain SGNF > females with pain. Responsiveness: SGNF $\downarrow$ in flexor \& extensor strength @ 30 months. SGNF larger $\downarrow$ in extensor strength @ 30 months in range of motion group @ $120^{\circ}$ /s, \& with baseline knee OA @ 60\% $/$ s, \& larger $\downarrow$ if painful at baseline @ 120\% /s. SGNF larger $\downarrow$ in flexor strength @ 30 months in range of motion group @ 60\% correlation between exercise session adherence \& flexor ( $r=0.32$ to 0.37 ) \& extensor ( $r=0.32$ to 0.35 ) strength (a) $60 \%$ s for the strength training group.

Miltner et al. Germans with bilateral knee Cybex 6000 dynamometer measured

(2002) [83] OA $(n=43$; mean age $=$ flexor \& extensor strength (PT \&

Responsiveness: SGNF $\uparrow$ in knee flexor \& extensor 67.0 y) assigned to unilateral work) @ 60,90,120, 150, \& 180\%/s. hyaluronic acid injections.

Ögüt Turk females with knee OA

(2018) [84] assigned to Kinesiology

Humac Norm isokinetic

Responsiveness: SGNF $\uparrow$ in knee extensor strength in Taping $\left(n=31\right.$; mean age $=$ strength $(\mathrm{PT}) @ 60 \& 180^{\circ} / \mathrm{s}$

53.8 y) or Sham Taping ( $n$

$=30$; mean age $=53.1 \mathrm{y}$ ) to

the extensor muscles.

Park et al. Korean females with knee measured knee extensor isokinetic exercise with whole body strength (PT) @ 60\% $/ \mathrm{s} \&$ isometric vibration $\left(n=11\right.$; mean age strength (PT) @ $60^{\circ}$.

$=62.5 \mathrm{y})$ or home based exercise without $(n=11$; mean age $=60.0 \mathrm{y}$ ).

Patsika et al. Greek females with knee OA Cybex dynamometer measured (2014) [86] $\quad(n=12$; mean age $=\quad$ concentric \& eccentric knee flexor \& 60.3 y) \& healthy controls ( $n$ extensor strength (flexor/extensor $=11$; mean age $=56.5 \mathrm{y})$. ratio) @ 60, $120, \& 150^{\circ} / \mathrm{s}$

Responsiveness: no difference in strength change between groups. SGNF $\uparrow$ right \& not left knee extensor strength @ 60\% $/ \mathrm{s}$. SGNF $\uparrow$ knee extensor isometric strength in both knees for both groups.

Validity: females with knee OA flexor/extensor ratio $\overline{\mathrm{SGNF}}>$ healthy controls. 
Table 1, continued

\begin{tabular}{|c|c|c|c|}
\hline $\begin{array}{l}\text { Authorship } \\
\text { (year) }\end{array}$ & Participants & Isokinetic procedures & Findings \\
\hline $\begin{array}{l}\text { Peeler and } \\
\text { Ripat } \\
(2018)[87]\end{array}$ & $\begin{array}{l}\text { Canadians with knee OA ( } n \\
=31 ; \text { mean age }=64.2 \text { y) } \\
\text { assigned to low-intensity } \\
\text { exercise. }\end{array}$ & $\begin{array}{l}\text { Biodex System } 3 \text { measured maximal } \\
\text { isokinetic knee extensor strength } \\
\text { (bodyweight adjusted PT) @ 60,180, } \\
\& 240^{\circ} \% \text { s. }\end{array}$ & $\begin{array}{l}\text { Validity: no difference in strength between affected \& } \\
\text { unaffected limbs at baseline. } \\
\text { Responsiveness: females had SGNF } \uparrow \text { in knee extensor } \\
\text { strength @ 60,180 \& } 240^{\circ} \% \text { s. }\end{array}$ \\
\hline $\begin{array}{l}\text { Peixoto et al. } \\
(2011)[88]\end{array}$ & $\begin{array}{l}\text { Older Brazilian females with } \\
\text { unilateral or bilateral knee } \\
\text { OA }(n=35 ; \text { mean age }= \\
73.3 \text { y). }\end{array}$ & $\begin{array}{l}\text { Biodex System } 3 \text { Pro dynamometer } \\
\text { measured concentric knee flexor \& } \\
\text { extensor strength (bodyweight } \\
\text { adjusted PT) @ } 60 \& 180^{\circ} / \mathrm{s} .\end{array}$ & $\begin{array}{l}\text { Validity: SGNF inverse correlation of knee extensor } \\
\text { strength @ } 180^{\circ} / \mathrm{s} \& \text { age }(r=-0.34) \text {, but not @ } 60^{\circ} / \mathrm{s} \text {. } \\
\text { No correlation with proprioceptive acuity \& strength. }\end{array}$ \\
\hline $\begin{array}{l}\text { Pelletier et al. } \\
\text { (2013) [89] }\end{array}$ & $\begin{array}{l}\text { Canadian females with knee } \\
\text { OA }(n=17 ; \text { mean age }= \\
60.3 \text { y) assigned power } \\
\text { training. }\end{array}$ & $\begin{array}{l}\text { Biodex System } 3 \text { dynamometer } \\
\text { measured knee extensor strength } \\
\text { (power \& work) @ } 180^{\circ} / \mathrm{s} \& \\
\text { isometric knee extensor strength (PT) } \\
\text { @ } 30^{\circ} \text {. }\end{array}$ & $\begin{array}{l}\text { Responsiveness: SGNF } \uparrow \text { knee extensor power \& work } \\
\text { but no difference in isometric strength. }\end{array}$ \\
\hline $\begin{array}{l}\text { Peloquin et al. } \\
\text { (1999) [90] }\end{array}$ & $\begin{array}{l}\text { Canadians with knee OA } \\
\text { assigned an exercise program } \\
(n=59 ; \text { mean age }=65.6 \\
\text { y) or educational control }(n \\
=65 ; \text { mean age }=66.4 \mathrm{y}) .\end{array}$ & $\begin{array}{l}\text { Isokinetic dynamometer measured } \\
\text { knee flexor \& extensor strength (PT) } \\
\text { @ } 30 \& 90^{\circ} / \mathrm{s} \& \text { isometric flexor \& } \\
\text { extensor strength (PT) @ } 30 \& 60^{\circ} \text {. }\end{array}$ & $\begin{array}{l}\text { Responsiveness: improvement in isometric knee } \\
\text { extensor, \& isokinetic \& isometric knee flexor strength in } \\
\text { exercise group was SGNF > control group. No between } \\
\text { group differences in isometric knee flexor strength. }\end{array}$ \\
\hline $\begin{array}{l}\text { Petrella et al. } \\
\text { (2017) [91] }\end{array}$ & $\begin{array}{l}\text { Brazilians with knee OA }(n \\
=24 \text { mean age }=52.4 \mathrm{y}) \& \\
\text { healthy controls }(n=20 ; \\
\text { mean age }=51.4 \mathrm{y})\end{array}$ & $\begin{array}{l}\text { Biodex System } 3 \text { measured concentric } \\
\& \text { eccentric knee extensor strength } \\
\text { (bodyweight adjusted PT) @ } 90^{\circ} / \mathrm{s}, \& \\
\text { isometric knee extensor strength } \\
\text { (bodyweight adjusted PT) @ } 60^{\circ} \text {. }\end{array}$ & $\begin{array}{l}\text { Validity: knee OA group eccentric knee extensor strength } \\
<\text { control group, but not concentric or isometric strength. }\end{array}$ \\
\hline $\begin{array}{l}\text { Pua et al. } \\
\text { (2011) [92] }\end{array}$ & $\begin{array}{l}\text { Singaporeans with knee OA } \\
\text { awaiting total knee } \\
\text { replacement }(n=104 \text {; mean } \\
\text { age }=67 \mathrm{y})\end{array}$ & $\begin{array}{l}\text { Biodex dynamometer measured } \\
\text { isometric knee extensor strength } \\
\text { (body weight adjusted PT) @ } 75^{\circ} \text {. }\end{array}$ & $\begin{array}{l}\text { Validity: variation in SF36 physical function \& gait } \\
\text { speed were partially explained }\left(r^{2}=0.32 \text { to } 0.55\right) \text { by } \\
\text { knee extensor strength, center of pressure displacement, } \\
\& \text { covariates. }\end{array}$ \\
\hline $\begin{array}{l}\text { Pua et al. } \\
(2013) \text { [93] }\end{array}$ & $\begin{array}{l}\text { Singaporeans with knee OA } \\
\text { awaiting total knee } \\
\text { replacement }(n=67 \text {; mean } \\
\text { age }=67.2 \mathrm{y})\end{array}$ & $\begin{array}{l}\text { Biodex dynamometer measured } \\
\text { isometric knee extensor strength } \\
\text { (body weight adjusted PT) @ } 75^{\circ} \text {. }\end{array}$ & $\begin{array}{l}\text { Validity: individuals with slower gait speeds }(<1.0 \mathrm{~m} / \mathrm{s}) \\
\text { had SGNF } \downarrow \text { knee extensor strength. }\end{array}$ \\
\hline $\begin{array}{l}\text { Robbins et al. } \\
\text { (2011) [94] }\end{array}$ & $\begin{array}{l}\text { Canadians with symptomatic } \\
\text { knee OA of medial } \\
\text { compartment }(n=38 \text {; mean } \\
\text { age }=54 \text { y) }\end{array}$ & $\begin{array}{l}\text { Biodex System } 3 \text { Pro dynamometer } \\
\text { measured concentric knee extensor } \\
\text { strength (PT)@ } 60^{\circ} / \mathrm{s} .\end{array}$ & $\begin{array}{l}\text { Validity: SGNF correlation of knee extensor strength } \\
\text { with KOOS pain score }(r=0.49) \text {. Variation in KOOS } \\
\text { pain score was partially explained }\left(r^{2}=0.49\right) \text { by BMI, } \\
\text { knee extensor strength, external knee adduction moment, } \\
\& \text { steps/day. }\end{array}$ \\
\hline $\begin{array}{l}\text { Rodrigues- } \\
\text { da-Silva et al. } \\
(2017)[95]\end{array}$ & $\begin{array}{l}\text { Brazilians with knee OA }(n \\
=130 ; \text { mean age }=64.3 \mathrm{y}) \\
\text { pre \& post-familiarization to } \\
\text { isokinetic testing. }\end{array}$ & $\begin{array}{l}\text { Biodex System } 3 \text { measured flexor \& } \\
\text { extensor isokinetic strength (PT, body } \\
\text { weight adjusted PT, total work, } \\
\text { coefficient of variation, \& } \\
\text { agonist-antagonist ratio) @ } 60^{\circ} / \mathrm{s} \text {. }\end{array}$ & $\begin{array}{l}\text { Responsiveness: SGNF } \uparrow \text { strength (PT, body weight } \\
\text { adjusted PT, \& total work) with } \downarrow \text { coefficient of variation } \\
\text { post-familiarization to isokinetic testing. No difference in } \\
\text { agonist-antagonist ratio. }\end{array}$ \\
\hline $\begin{array}{l}\text { Rodrigues da } \\
\text { Silva } \\
(2019)[96]\end{array}$ & $\begin{array}{l}\text { Brazilians with knee OA }(n \\
=136 ; \text { mean age }=66.3 \mathrm{y}) \\
\text { assigned interdisciplinary } \\
\text { exercise education program. }\end{array}$ & $\begin{array}{l}\text { Biodex System } 3 \text { dynamometer } \\
\text { measured concentric knee flexor \& } \\
\text { extensor strength (PT, bodyweight } \\
\text { adjusted PT, total work, coefficient of } \\
\text { variation, \& agonist-antagonist ratio) } \\
\text { @ } 60^{\circ} / \mathrm{s} \text {. }\end{array}$ & $\begin{array}{l}\text { Responsiveness: SGNF } \uparrow \text { knee flexor \& extensor } \\
\text { strength (all measures) \& } \downarrow \text { coefficient of variation after } \\
\text { training. No difference in agonist-antagonist ratio or } \\
\text { strength between legs. }\end{array}$ \\
\hline $\begin{array}{l}\text { Røgind et al. } \\
\text { (1998) [97] }\end{array}$ & $\begin{array}{l}\text { Danes with knee OA } \\
\text { assigned to exercise }(n= \\
11 ; \text { mean age }=69.3 \mathrm{y}) \text { or } \\
\text { control }(n=12 ; \text { mean age } \\
=73.0 \mathrm{y})\end{array}$ & $\begin{array}{l}\text { Cybex } 6000 \text { dynamometer measured } \\
\text { knee flexor \& extensor strength (PT) } \\
@ 30,60,90 \& 120^{\circ} / \mathrm{s} \& \text { isometric @ } \\
90^{\circ} .\end{array}$ & $\begin{array}{l}\text { Responsiveness: SGNF } \uparrow \text { knee flexor \& extensor } \\
\text { strength @ } 30^{\circ} / \mathrm{s} \& \text { isometric for exercise group. }\end{array}$ \\
\hline $\begin{array}{l}\text { Samut et al. } \\
(2015)[98]\end{array}$ & $\begin{array}{l}\text { Turks with knee OA } \\
\text { assigned to isokinetic } \\
\text { exercise }(n=15 ; \text { mean age } \\
=62.5 \mathrm{y}), \text { aerobic exercise } \\
(n=14 ; \text { mean age }= \\
57.6 \mathrm{y}), \text { or control }(n=13 ; \\
\text { mean age }=60.9 \mathrm{y}) .\end{array}$ & $\begin{array}{l}\text { Biodex System } 3 \text { Pro dynamometer } \\
\text { measured concentric knee flexor \& } \\
\text { extensor strength (bodyweight } \\
\text { adjusted PT) @ } 60 \& 180^{\circ} \% \text { s. }\end{array}$ & $\begin{array}{l}\text { Responsiveness: SGNF } \uparrow \text { in right knee extensor strength } \\
\text { in aerobic exercise group, but not other groups or the left } \\
\text { knee for all groups. SGNF } \uparrow \text { in left knee flexor strength } \\
@ 60^{\circ} / \mathrm{s} \text { in isokinetic group, \& @ } 180^{\circ} / \mathrm{s} \text { in aerobic } \\
\text { exercise group, with no difference in right knee strength } \\
\text { in all groups. }\end{array}$ \\
\hline
\end{tabular}


Table 1, continued

\begin{tabular}{|c|c|c|c|}
\hline $\begin{array}{l}\text { Authorship } \\
\text { (year) }\end{array}$ & Participants & Isokinetic procedures & Findings \\
\hline $\begin{array}{l}\text { Sanchez- } \\
\text { Ramirez et al. } \\
\text { (2013) [99] }\end{array}$ & $\begin{array}{l}\text { Dutch with knee OA }(n= \\
284 ; \text { mean age }=61.5 \mathrm{y})\end{array}$ & $\begin{array}{l}\text { EnKnee dynamometer measured knee } \\
\text { flexor \& extensor strength (body } \\
\text { weight adjusted PT) @ } 60^{\circ} / \mathrm{s} .\end{array}$ & $\begin{array}{l}\text { Validity: SGNF association in one leg stance time ( }> \\
30 \text { s) with knee strength }(\beta=0.1 \text { to } 0.23 \text { ). }\end{array}$ \\
\hline $\begin{array}{l}\text { Sanchez- } \\
\text { Ramirez et al. } \\
\text { (2013) [100] }\end{array}$ & $\begin{array}{l}\text { Dutch with knee OA }(n= \\
285 ; \text { mean age }=61.7 \mathrm{y})\end{array}$ & $\begin{array}{l}\text { EnKnee dynamometer measured knee } \\
\text { flexor \& extensor strength (body } \\
\text { weight adjusted PT) @ } 60^{\circ} / \mathrm{s} .\end{array}$ & $\begin{array}{l}\text { Validity: SGNF association of lower muscle strength } \\
\text { with elevated C-reactive protein }(\beta=-0.10) \& \\
\text { erythrocyte sedimentation rate }(\beta=-0.12) \text { which was } \\
\text { no longer SGNF after controlling for BMI. }\end{array}$ \\
\hline $\begin{array}{l}\text { Sanchez- } \\
\text { Ramirez et al. } \\
\text { (2014) [101] }\end{array}$ & $\begin{array}{l}\text { Dutch with knee OA }(n= \\
186 ; \text { mean age }=61.0 \mathrm{y})\end{array}$ & $\begin{array}{l}\text { EnKnee dynamometer measured knee } \\
\text { flexor \& extensor strength } \\
\text { (bodyweight adjusted PT) @ } 60 \% \text { s. }\end{array}$ & $\begin{array}{l}\text { Responsiveness: group with elevated c-reactive protein } \\
\text { @ baseline \& 2y had SGNF < strength improvements } \\
(\beta=-0.21 \text { to }-0.17) @ 2 \text {-years. }\end{array}$ \\
\hline $\begin{array}{l}\text { Sanchez- } \\
\text { Ramirez et al. } \\
\text { (2015) [102] }\end{array}$ & $\begin{array}{l}\text { Dutch with knee OA }(n= \\
186 ; \text { mean age }=61.2 \mathrm{y})\end{array}$ & $\begin{array}{l}\text { EnKnee dynamometer measured knee } \\
\text { flexor \& extensor strength } \\
\text { (bodyweight adjusted PT) @ 60\% } / \mathrm{s} \text {. }\end{array}$ & $\begin{array}{l}\text { Responsiveness: SGNF } \uparrow \text { in mean knee muscle, } \& \text { flexor } \\
\& \text { extensor strength @ 2-years. SGNF association } \\
\text { between knee extensor strength \& WOMAC physical } \\
\text { function score }(\beta=-2.8), \text { Get Up and Go Test }(\beta= \\
-0.08), \& 12 \text {-Stairs Step Test }(\beta=-2.7) \text {. }\end{array}$ \\
\hline $\begin{array}{l}\text { Santos et al. } \\
\text { (2011) [103] }\end{array}$ & $\begin{array}{l}\text { Brazilian females with knee } \\
\text { OA }(n=80 ; \text { mean age }= \\
71.2 \text { y). }\end{array}$ & $\begin{array}{l}\text { Biodex System } 3 \text { Pro dynamometer } \\
\text { measured concentric knee flexor \& } \\
\text { extensor strength (bodyweight } \\
\text { adjusted PT) } 60 \& 180^{\circ} \% \text { s. }\end{array}$ & $\begin{array}{l}\text { Validity: SGNF inverse correlation of Interleukin- } 6 \text { with } \\
\text { knee flexor strength }(r=-0.23) \& \text { hamstring to } \\
\text { quadriceps ratio }(r=0.25) @ 180^{\circ} / \mathrm{s} \text {. No correlation of } \\
\text { knee extensor strength with I'nterleukin- } 6 \text {. }\end{array}$ \\
\hline $\begin{array}{l}\text { Schilke et al. } \\
\text { (1996) [104] }\end{array}$ & $\begin{array}{l}\text { Americans with knee OA } \\
\text { assigned to isokinetic } \\
\text { exercise }(n=10 \text {; mean age } \\
=64.5 \mathrm{y}) \text { or control }(n= \\
10 ; \text { mean age }=68.4 \mathrm{y})\end{array}$ & $\begin{array}{l}\text { Cybex II dynamometer measured } \\
\text { flexor \& extensor strength (PT) @ } \\
90^{\circ} / \mathrm{s} \text {. }\end{array}$ & $\begin{array}{l}\text { Responsiveness: SGNF } \uparrow \text { flexor \& extensor strength in } \\
\text { exercise group, \& right flexors \& left extensors in control } \\
\text { group. Strength } \uparrow \text { for exercise group in left flexor } \\
\text { strength SGNF }>\text { control group. No other between group } \\
\text { differences in knee strength. }\end{array}$ \\
\hline $\begin{array}{l}\text { Segal et al. } \\
\text { (2009) [105] }\end{array}$ & $\begin{array}{l}\text { Americans (mean age }= \\
62.4 \text { y) with knee OA }(n= \\
2078) \& \text { without knee OA }(n \\
=1617)\end{array}$ & $\begin{array}{l}\text { Cybex } 350 \text { dynamometer measured } \\
\text { concentric knee flexor \& extensor } \\
\text { strength (PT) @ 60\% } / \text { s. }\end{array}$ & $\begin{array}{l}\text { Validity: knee extensor strength in males SGNF }>\text { fe- } \\
\text { males. } \\
\text { Responsiveness: knee strength was not predictive of } \\
\text { incident radiographic OA @ } 30 \text { months. SGNF } \downarrow \text { risk of } \\
\text { incident symptomatic knee OA for highest tertile in } \\
\text { females (OR }=0.4) \& \text { males }(\mathrm{OR}=0.5) \text {. }\end{array}$ \\
\hline $\begin{array}{l}\text { Segal et al. } \\
(2009)[106]\end{array}$ & $\begin{array}{l}\text { Americans with knee OA or } \\
\text { at risk for knee OA }(n= \\
1269 \text { female knees } \& 1006 \\
\text { male knees; mean age }= \\
62.2 \mathrm{y})\end{array}$ & $\begin{array}{l}\text { Cybex } 350 \text { dynamometer measured } \\
\text { concentric knee flexor \& extensor } \\
\text { strength (PT) @ } 60^{\circ} / \mathrm{s} \text {. }\end{array}$ & $\begin{array}{l}\text { Reliability: knee strength test-retest }(\mathrm{ICC}=0.94) \text {. } \\
\text { Responsiveness: concentric knee extensor strength does } \\
\text { not predict development of knee symptoms @ } 15 \text { or } \\
30 \text { months. }\end{array}$ \\
\hline $\begin{array}{l}\text { Segal et al. } \\
\text { (2010) [107] }\end{array}$ & $\begin{array}{l}\text { Americans with knee OA or } \\
\text { risk factors for knee OA }(n \\
=2856 ; \text { mean age }= \\
62.2 \text { y). }\end{array}$ & $\begin{array}{l}\text { Cybex } 350 \text { dynamometer measured } \\
\text { knee flexor \& extensor strength (PT) } \\
@ 60^{\circ} / \mathrm{s} \text {. }\end{array}$ & $\begin{array}{l}\text { Validity: male knee flexor \& extensor strength SGNF }> \\
\text { females. } \\
\text { Reliability: test-retest knee strength (ICC }=0.94) . \\
\text { Responsiveness: females in the lowest tertile of extensor } \\
\text { strength @ baseline SGNF }>\text { risk of joint space } \\
\text { narrowing @ } 30 \text { months for the whole knee }(\mathrm{OR}=1.66) \\
\text { or tibiofemoral joint }(\mathrm{OR}=1.69) \text {. No difference in joint } \\
\text { space narrowing based upon strength for males. }\end{array}$ \\
\hline $\begin{array}{l}\text { Segal et al. } \\
\text { (2011) [108] }\end{array}$ & $\begin{array}{l}\text { Americans who were } \\
\text { non-obese }(n=113: \text { mean } \\
\text { age }=55.0 \mathrm{y}) \text {, obese }(n= \\
101 ; \text { mean age }=54.7 \mathrm{y}), \text { or } \\
\text { moderate to severely obese } \\
(n=89 ; \text { mean age }= \\
55.2 \mathrm{y})\end{array}$ & $\begin{array}{l}\text { Biodex System } 3 \text { dynamometer } \\
\text { measured knee extensor strength (PT) } \\
\text { @ } 60^{\circ} / \mathrm{s} \text {. }\end{array}$ & $\begin{array}{l}\text { Validity: No difference in strength by BMI or presence } \\
\text { of knee OA. }\end{array}$ \\
\hline $\begin{array}{l}\text { Serrão et al. } \\
\text { (2012) [109] }\end{array}$ & $\begin{array}{l}\text { Brazilians with knee OA }(n \\
=27 \text {; mean age }=51.8 \mathrm{y})\end{array}$ & $\begin{array}{l}\text { Biodex System } 3 \text { dynamometer } \\
\text { measured concentric \& eccentric knee } \\
\text { extensor strength (body weight } \\
\text { adjusted PT) @ } 90^{\circ} / \mathrm{s} \text {. }\end{array}$ & $\begin{array}{l}\text { Validity: SGNF inverse correlation of concentric \& } \\
\text { eccentric extensor strength with WOMAC pain }(r= \\
-0.70 \& r=-0.56) \text {, stiffness }(r=-0.62 \& r= \\
-0.44), \& \text { physical function }(r=-0.54 \&-0.52)\end{array}$ \\
\hline $\begin{array}{l}\text { Serrão et al. } \\
\text { (2015) [110] }\end{array}$ & $\begin{array}{l}\text { Brazilian males with knee } \\
\text { OA }(n=22 ; \text { mean age }= \\
51.9 \text { y) or without knee OA } \\
(n=18 ; \text { mean age }= \\
52.0 \mathrm{y})\end{array}$ & $\begin{array}{l}\text { Biodex System } 3 \text { dynamometer } \\
\text { measured concentric \& eccentric knee } \\
\text { flexor \& extensor strength } \\
\text { (bodyweight adjusted PT) @ } 90 \& \\
180^{\circ} / \mathrm{s} \text {. }\end{array}$ & $\begin{array}{l}\text { Validity: eccentric knee extensor strength in group } \\
\text { without knee OA SGNF }>\text { knee OA group. OA group } \\
\text { knee extensor strength to Type } 2 \mathrm{~b} \& \text { Type } 1 \text { fiber cross } \\
\text { sectional area SGNF }<\text { control group. No between group } \\
\text { difference in concentric knee strength or muscle fiber } \\
\text { cross sectional area. }\end{array}$ \\
\hline
\end{tabular}


Table 1, continued

\begin{tabular}{|c|c|c|c|}
\hline $\begin{array}{l}\text { Authorship } \\
\text { (year) }\end{array}$ & Participants & Isokinetic procedures & Findings \\
\hline $\begin{array}{l}\text { Shakoor et al. } \\
\text { (2017) [111] }\end{array}$ & $\begin{array}{l}\text { Americans with knee OA or } \\
\text { at high risk for knee OA }(n \\
=1803 ; \text { mean age }= \\
67.6 \mathrm{y})\end{array}$ & $\begin{array}{l}\text { Cybex } 350 \text { dynamometer measured } \\
\text { isokinetic extensor strength } \\
\text { (bodyweight adjusted PT) @ } 60^{\circ} / \mathrm{s} \text {. }\end{array}$ & $\begin{array}{l}\text { Responsiveness: } \uparrow \text { Strength associated with SGNF } \downarrow \text { risk } \\
\text { of incident }(\mathrm{RR}=0.48) \& \text { worsening }(\mathrm{RR}=0.64) \text { of } \\
\text { slipping \& shifting symptoms; } \downarrow \text { incident }(\mathrm{RR}=0.86) \& \\
\text { worsening }(\mathrm{RR}=0.85) \text { of buckling, } \downarrow \text { instability } \\
\text { symptoms }(\mathrm{RR}=0.53) \& \text { worsening }(\mathrm{RR}=0.73) .\end{array}$ \\
\hline $\begin{array}{l}\text { Silva et al. } \\
(2018)[112]\end{array}$ & $\begin{array}{l}\text { Brazilians with knee OA } \& \\
\text { sleep apnea }(n=15 ; \text { mean } \\
\text { age }=55.4 \mathrm{y}), \text { sleep apnea } \\
\text { without knee OA }(n=15 ; \\
\text { mean age }=54.9 \mathrm{y}), \text { knee } \\
\text { OA without sleep apnea }(n \\
=15 ; \text { mean age }=53.2 \mathrm{y}), \\
\text { or control }(n=15 \text { mean age } \\
=52.6 \mathrm{y}) .\end{array}$ & $\begin{array}{l}\text { Biodex System } 3 \text { measured isometric } \\
\text { knee extensor strength (bodyweight } \\
\text { adjusted PT) @ } 60^{\circ}, \text { \& concentric \& } \\
\text { eccentric knee extensor strength } \\
\text { (bodyweight adjusted PT) @ } 90 \& \text { \& } \\
180^{\circ} \% \text { s. }\end{array}$ & $\begin{array}{l}\text { Validity: concentric knee extensor strength @ } 90 \text { \& } \\
180^{\circ} / \mathrm{s} \text { in group with knee OA \& sleep apnea SGNF }< \\
\text { participants without knee OA, \& group with knee OA } \\
\text { without sleep apnea SGNF }<\text { groups without knee OA. } \\
\text { Isometric knee extensor strength in group with knee OA } \\
\& \text { sleep apnea SGNF }<\text { participants without knee OA, \& } \\
\text { group with knee OA without sleep apnea SGNF }< \\
\text { groups without knee OA. No differences in eccentric } \\
\text { knee extensor strength between groups. }\end{array}$ \\
\hline $\begin{array}{l}\text { Skou et al. } \\
(2016)[113]\end{array}$ & $\begin{array}{l}\text { Americans with knee OA }(n \\
=1257 ; \text { mean age }= \\
62.2 \mathrm{y})\end{array}$ & $\begin{array}{l}\text { Cybex } 350 \text { dynamometer measured } \\
\text { knee extensor strength (PT) @ 60\% }\end{array}$ & $\begin{array}{l}\text { Validity: no difference in risk for TKA by knee extensor } \\
\text { strength when adjusted for radiographic severity. }\end{array}$ \\
\hline $\begin{array}{l}\text { Slemenda et } \\
\text { al. } \\
(1997)[114]\end{array}$ & $\begin{array}{l}\text { Americans with knee OA }(n \\
=112 ; \text { mean age }=72.4 \mathrm{y}) \\
\text { or without knee OA }(n= \\
350 ; \text { mean age }=72.1 \mathrm{y})\end{array}$ & $\begin{array}{l}\text { Kin-Com } 500 \mathrm{H} \text { dynamometer } \\
\text { measured concentric \& eccentric knee } \\
\text { flexor \& extensor strength }(\mathrm{PT}) @ 60 \\
\& 120^{\circ} / \mathrm{s} \text {. }\end{array}$ & $\begin{array}{l}\text { Validity: knee extensor strength in knee OA group SGNF } \\
<\text { group without knee OA. Knee extensor strength in } \\
\text { females with knee OA but no pain group SGNF }>\text { knee } \\
\text { OA with pain group. Risk of radiographic }(\mathrm{OR}=0.80) \\
\& \text { symptomatic }(\mathrm{OR}=0.71) \text { knee OA SGNF } \downarrow \text { per } 10 \\
\text { lb/ft of knee extensor strength. }\end{array}$ \\
\hline $\begin{array}{l}\text { Slemenda et } \\
\text { al. } \\
\text { (1998) [115] }\end{array}$ & $\begin{array}{l}\text { Elderly Americans (mean } \\
\text { age }=70.8 \text { y) with knee OA } \\
(n=99) \text { or without knee } \\
\text { OA }(n=237)\end{array}$ & $\begin{array}{l}\text { Kin-Com } 500 \mathrm{H} \text { dynamometer } \\
\text { measured concentric knee flexor \& } \\
\text { extensor strength (PT, body weight } \\
\text { adjusted PT, \& lower extremity } \\
\text { muscle mass adjusted PT) @ } 60^{\circ} / \mathrm{s} .\end{array}$ & $\begin{array}{l}\text { Validity: knee strength (body weight adjusted) in females } \\
\text { with knee OA SGNF }<\text { controls. No difference in relative } \\
\text { strength by knee OA status for males. SGNF correlation } \\
\text { between body weight \& knee extensor strength in male } \\
\text { controls ( } r=0.22 \text { ) but not females. } \\
\text { Responsiveness: SGNF correlation between knee } \\
\text { extensor strength \& body weight ( } r=-0.74 \text { ) among } \\
\text { females with incident knee OA. SGNF correlation } \\
\text { between body weight \& knee extensor strength (adjusted } \\
\text { for lower extremity muscle mass) in females controls ( } r \\
=-0.21 \text { ), with incident knee OA ( } r=-0.83 \text { ), \& those } \\
\text { with knee OA who did not develop contralateral knee } \\
\text { OA ( } r=-0.46 \text { ). No correlation between knee extensor } \\
\text { strength (adjusted for lower extremity muscle mass) \& } \\
\text { body weight in males. No between group difference in } \\
\text { knee flexor strength for controls or incident knee OA. }\end{array}$ \\
\hline $\begin{array}{l}\text { Stefanik et al. } \\
(2011)[116]\end{array}$ & $\begin{array}{l}\text { Americans with knee OA or } \\
\text { at risk for knee OA }(n=807 \\
\text { knees; mean age }=62 \mathrm{y})\end{array}$ & $\begin{array}{l}\text { Cybex } 350 \text { dynamometer measured } \\
\text { concentric knee extensor strength } \\
\text { (bodyweight adjusted PT \& BMI } \\
\text { adjusted PT) @ 60\% }\end{array}$ & $\begin{array}{l}\text { Validity: individuals in the lowest tertile of knee exten- } \\
\text { sor strength had SGNF } \uparrow \text { medial \& lateral patellofemoral } \\
\text { joint cartilage damage \& lateral patellofemoral bone mar- } \\
\text { row lesions. } \\
\text { Reliability: concentric knee extensor strength test-retest } \\
(\mathrm{ICC}=0.94) \text {. }\end{array}$ \\
\hline $\begin{array}{l}\text { Tan et al. } \\
(1995)[117]\end{array}$ & $\begin{array}{l}\text { Turkish females with } \\
\text { radiographic knee OA \& } \\
\text { knee pain }(n=30 ; \text { mean age } \\
=63.0 \mathrm{y}), \text { knee pain without } \\
\text { radiographic knee OA }(n= \\
30 ; \text { mean age }=51.9 \mathrm{y}), \& \\
\text { healthy controls }(n=30 ; \\
\text { mean age }=50.6 \mathrm{y}) .\end{array}$ & $\begin{array}{l}\text { Cybex-350 dynamometer measured } \\
\text { knee flexor \& extensor strength (PT, } \\
\text { \& agonist/antagonist ratio) @ } 60 \& \\
180^{\circ} / \mathrm{s}, \& \text { isometric flexor \& extensor } \\
\text { strength (PT \& agonist/antagonist } \\
\text { ratio)@ } 30 \& 60^{\circ} \text {. }\end{array}$ & $\begin{array}{l}\text { Validity: knee flexor \& extensor isometric \& isokinetic } \\
\text { strength in both OA groups SGNF }<\text { healthy controls. } \\
\text { No between group differences in agonist/antagonist ratio. }\end{array}$ \\
\hline $\begin{array}{l}\text { Tang et al. } \\
(2005)[118]\end{array}$ & $\begin{array}{l}\text { Taiwanese with bilateral } \\
\text { knee OA }(n=25 ; \text { mean age } \\
=61.3 \mathrm{y}) \text { assigned } \\
\text { hyaluronan injection to each } \\
\text { knee. }\end{array}$ & $\begin{array}{l}\text { Kin-Com dynamometer measured } \\
\text { knee flexor \& extensor strength } \\
\text { (bodyweight adjusted PT) @ } 80 \& \\
240 \% \text { s. }\end{array}$ & $\begin{array}{l}\text { Responsiveness: SGNF } \uparrow \text { concentric \& eccentric flexor } \\
\text { \& extensor strength @ } 80 \& 240^{\circ} / \mathrm{s} \text {, except non-SGNF } \uparrow \\
\text { in concentric flexor strength @ } 240^{\circ} / \mathrm{s} \text {. }\end{array}$ \\
\hline
\end{tabular}


Table 1, continued

\begin{tabular}{|c|c|c|c|}
\hline $\begin{array}{l}\text { Authorship } \\
\text { (year) }\end{array}$ & Participants & Isokinetic procedures & Findings \\
\hline $\begin{array}{l}\text { Tok et al. } \\
\text { (2011) [119] }\end{array}$ & $\begin{array}{l}\text { Turks with knee OA } \\
\text { assigned to receive } \\
\text { conventional physical } \\
\text { therapy with combined } \\
\text { continuous passive motion } \& \\
\text { electrical stimulation }(n= \\
20 ; \text { mean age }=61.8 \mathrm{y}) \text { or } \\
\text { conventional physical } \\
\text { therapy with isometric } \\
\text { exercise }(n=20 ; \text { mean age } \\
=66.6 \mathrm{y})\end{array}$ & $\begin{array}{l}\text { Cybex } 6000 \text { dynamometer measured } \\
\text { concentric \& eccentric flexor \& } \\
\text { extensor strength (PT \& work) @ } \\
60^{\circ} / \mathrm{s} \text {. }\end{array}$ & $\begin{array}{l}\text { Responsiveness: SGNF improvement in right flexor } \\
\text { strength but not left or knee extension strength for group } \\
\text { receiving continuous passive motion \& electrical } \\
\text { stimulation. }\end{array}$ \\
\hline Tuna and & Turkish females with knee & Cybex NORM 6000 measured knee & Validity: no difference in extensor strength @ 90\%/s \\
\hline $\begin{array}{l}\text { Balc1 } \\
\text { (2014) }[120]\end{array}$ & $\begin{array}{l}\text { OA }(n=117 ; \text { mean age }= \\
57.9 \mathrm{y})\end{array}$ & $\begin{array}{l}\text { flexor \& extensor strength (PT, work) } \\
\text { @ } 90 \& 180^{\circ} / \mathrm{s} \text {. }\end{array}$ & $\begin{array}{l}\text { based upon presence or absence of radiographic knee } \\
\text { OA. Knee flexor work on left limb only SGNF }<\text { right } \\
\text { limb. }\end{array}$ \\
\hline $\begin{array}{l}\text { Tuna et al. } \\
\text { (2016) [121] }\end{array}$ & $\begin{array}{l}\text { Turks with knee OA }(n= \\
40, \text { mean age }=52.03 \text { y) } \\
\text { undergoing strength training } \\
\text { protocol. }\end{array}$ & $\begin{array}{l}\text { Cybex NORM } 6000 \text { measured knee } \\
\text { flexor \& extensor strength (PT \& } \\
\text { work) @ } 60 \& 180^{\circ} / \mathrm{s}, \& \text { isometric } \\
\text { strength (PT) @ } 30 \& 60^{\circ} .\end{array}$ & $\begin{array}{l}\text { Validity: SGNF correlation between cartilage thickness } \\
\& \text { isometric knee flexor }(r=0.25 \text { to } 0.30) \& \text { extensor } \\
(r=0.25) \text { strength @ } 30^{\circ} \& \text { isokinetic work @ } 180^{\circ} / \mathrm{s} \\
(r=0.25 \text { to } 0.32) \text { at baseline. No correlation between } \\
\text { cartilage thickness \& isokinetic PT or isometric strength } \\
@ 60^{\circ} \text { at baseline. } \\
\text { Responsiveness: knee flexor \& extensor PT @ } 30 \& \\
60^{\circ} / \mathrm{s} \& \text { total work @ } 180^{\circ} / \mathrm{s} \text { SGNF } \uparrow @ 1 \& 3 \text { months. } \\
\text { No correlation between cartilage thickness \& strength @ } \\
1 \text { month. SGNF correlation between cartilage thickness } \\
\& \text { isometric knee flexor strength }(r=0.39) \text {, isokinetic } \\
\text { knee flexor }(r=0.47) \& \text { extensor strength }(r=0.49) @ \\
60^{\circ} / \mathrm{s} \& \text { knee flexor }(r=0.38 \text { to } 0.40) \& \text { extensor }(r= \\
0.40 \text { to } 0.42) \text { work @ } 180^{\circ} / \mathrm{s} \text {. }\end{array}$ \\
\hline $\begin{array}{l}\text { Valtonen et al. } \\
\text { (2015) [122] }\end{array}$ & $\begin{array}{l}\text { Finns with late stage knee } \\
\text { OA }(n=56 ; \text { mean age }= \\
65.7 \mathrm{y})\end{array}$ & $\begin{array}{l}\text { Humac NORM dynamometer } \\
\text { measured knee flexor \& extensor } \\
\text { strength (PT \& power) @ } 60 \& \\
180^{\circ} / \mathrm{s} \text {. }\end{array}$ & $\begin{array}{l}\text { Validity: knee strength on affected knee SGNF }< \\
\text { unaffected knee. Variability in maximal walking speed } \\
\left(r^{2}=0.41 \text { to } 0.53\right) \& \text { stair ascension time }\left(r^{2}=0.58 \text { to }\right. \\
0.59) \text { was partially explained by asymmetry in knee } \\
\text { extensor power, contralateral or ipsilateral knee flexor \& } \\
\text { extensor power, \& WOMAC pain subscale. }\end{array}$ \\
\hline $\begin{array}{l}\text { van der Esch } \\
\text { et al. } \\
\text { (2006) [123] }\end{array}$ & $\begin{array}{l}\text { Dutch with knee OA }(n= \\
86 ; \text { mean age }=63.6 \mathrm{y})\end{array}$ & $\begin{array}{l}\text { EnKnee dynamometer measured knee } \\
\text { flexor \& extensor strength } \\
\text { (bodyweight adjusted PT) @ } 60 \% \text { s. }\end{array}$ & $\begin{array}{l}\text { Validity: SGNF correlation between knees for extensor } \\
\text { strength }(r=0.79) \text {, flexor strength }(r=0.83), \& \\
\text { average flexor/extensor strength }(r=0.85) \text {. SGNF } \\
\text { correlation for joint laxity with total muscle strength }(r \\
=-0.34) \text {. SGNF correlation for total muscle strength } \\
\text { with walking time }(r=-0.50), \& \text { WOMAC physical } \\
\text { function score }(r=-0.61) \text {. Variation in walking time } \\
\left(r^{2}=0.35\right) \& \text { WOMAC physical function score }\left(r^{2}=\right. \\
0.43) \text { were partially explained by muscle strength, \& } \\
\text { joint laxity. }\end{array}$ \\
\hline $\begin{array}{l}\text { van der Esch } \\
\text { et al. } \\
\text { (2007) [124] }\end{array}$ & $\begin{array}{l}\text { Dutch with symptomatic } \\
\text { knee OA }(n=63 ; \text { mean age } \\
=60 \mathrm{y})\end{array}$ & $\begin{array}{l}\text { EnKnee dynamometer measured knee } \\
\text { flexor \& extensor strength } \\
\text { (bodyweight adjusted PT) @ 60\% } / \mathrm{s} \text {. }\end{array}$ & $\begin{array}{l}\text { Validity: SGNF correlation between limbs for knee } \\
\text { extensor }(r=0.80) \& \text { flexor }(r=0.90) \text { strength, \& } \\
\text { average of flexor \& extensor strength }(r=0.94) \text {. } \\
\text { Variation in walking time can be partially explained } \\
\left(r^{2}=0.54\right) \text { by knee muscle strength, proprioception, \& } \\
\text { their interaction. Muscle strength is SGNF associated } \\
\text { with walking time, Get Up and Go Test, \& WOMAC } \\
\text { physical function subscale. }\end{array}$ \\
\hline $\begin{array}{l}\text { van der Esch } \\
\text { et al. } \\
\text { (2008) [125] }\end{array}$ & $\begin{array}{l}\text { Dutch with knee OA }(n= \\
63 ; \text { mean age }=60 \mathrm{y})\end{array}$ & $\begin{array}{l}\text { EnKnee dynamometer measured knee } \\
\text { flexor \& extensor strength } \\
\text { (bodyweight adjusted PT) @ } 60 \% \text { s. }\end{array}$ & $\begin{array}{l}\text { Validity: No correlation of varus-valgus range of motion } \\
\text { or mid-stance position with muscle strength. }\end{array}$ \\
\hline
\end{tabular}


Table 1, continued

\begin{tabular}{|c|c|c|c|}
\hline $\begin{array}{l}\text { Authorship } \\
\text { (year) }\end{array}$ & Participants & Isokinetic procedures & Findings \\
\hline $\begin{array}{l}\text { van der Esch } \\
\text { et al. } \\
(2008)[126]\end{array}$ & $\begin{array}{l}\text { Dutch with knee OA }(n= \\
63 ; \text { mean age }=60 \mathrm{y})\end{array}$ & $\begin{array}{l}\text { EnKnee dynamometer measured knee } \\
\text { flexor \& extensor strength } \\
\text { (bodyweight adjusted PT) @ } 60^{\circ} / \mathrm{s} \text {. }\end{array}$ & $\begin{array}{l}\text { Validity: Variation in walking time, Get Up and Go Test, } \\
\text { \& WOMAC physical function scores were partially } \\
\left.\text { explained ( } r^{2}=0.36 \text { to } 0.54\right) \text { by mid-stance } \\
\text { varus-valgus position, muscle strength, \& their } \\
\text { interaction. }\end{array}$ \\
\hline $\begin{array}{l}\text { van der Esch } \\
\text { et al. } \\
(2012)[127]\end{array}$ & $\begin{array}{l}\text { Dutch with knee OA }(n= \\
248, \text { mean age }=61.0 \mathrm{y})\end{array}$ & $\begin{array}{l}\text { EnKnee dynamometer measured knee } \\
\text { flexor \& extensor muscle strength } \\
\text { (body weight adjusted PT) @ } 60^{\circ} / \mathrm{s} \text {. }\end{array}$ & $\begin{array}{l}\text { Validity: knee strength \& pain do not moderate the } \\
\text { association of self-reported knee instability with activity } \\
\text { limitations. }\end{array}$ \\
\hline $\begin{array}{l}\text { Wageck et al. } \\
(2016)[128]\end{array}$ & $\begin{array}{l}\text { Brazilians with knee OA } \\
\text { assigned to kinesiotape }(n= \\
38 ; \text { mean age }=69.6 \text { y) or } \\
\text { sham taping }(n=38 ; \text { mean } \\
\text { age }=68.6 \text { y). }\end{array}$ & $\begin{array}{l}\text { Biodex System } 4 \text { dynamometer } \\
\text { measured knee flexor \& extensor } \\
\text { strength (bodyweight adjusted PT) @ } \\
60^{\circ} / \mathrm{s} \text {. }\end{array}$ & $\begin{array}{l}\text { Responsiveness: no difference in knee flexor \& extensor } \\
\text { strength between groups. }\end{array}$ \\
\hline $\begin{array}{l}\text { Weng et al. } \\
(2009) \text { [129] }\end{array}$ & $\begin{array}{l}\text { Taiwanese with bilateral } \\
\text { knee OA (mean age }= \\
64.0 \text { y) assigned to } \\
\text { non-exercise control ( } n= \\
33), \text { isokinetic strengthening } \\
(n=33) \text {, isokinetic } \\
\text { strengthening with static } \\
\text { stretching ( } n=33), \text { or } \\
\text { isokinetic strengthening with } \\
\text { proprioceptive } \\
\text { neuromuscular facilitation } \\
(n=33) \text {. }\end{array}$ & $\begin{array}{l}\text { Kin-Com } 505 \text { dynamometer } \\
\text { measured concentric \& eccentric knee } \\
\text { flexor \& extensor strength (PT) @ } 60 \\
\& 180^{\circ} / \mathrm{s} \text {. }\end{array}$ & $\begin{array}{l}\text { Responsiveness: SGNF } \uparrow \text { in knee flexor \& extensor } \\
\text { strength with isokinetic exercise (proprioceptive } \\
\text { neuromuscular facilitation }>\text { static stretching }> \\
\text { isokinetic training alone). }\end{array}$ \\
\hline $\begin{array}{l}\text { Wessel et al. } \\
(1996) \text { [130] }\end{array}$ & $\begin{array}{l}\text { Canadian females with knee } \\
\text { OA }(n=17 ; \text { mean age }= \\
60.8 \text { y) or without }(n=17 \\
\text { mean age }=63.8 \text { y) knee } \\
\text { OA. }\end{array}$ & $\begin{array}{l}\text { Kin-Com dynamometer measured } \\
\text { isokinetic knee extensor strength (PT) } \\
\text { @ 30,60, \& } 90^{\circ} / \mathrm{s} \text {. }\end{array}$ & $\begin{array}{l}\text { Validity: knee extensor strength in worse knee SGNF } \\
<\text { less affected limb in presence of OA. Knee extensor } \\
\text { strength @ } 60^{\circ} / \mathrm{s} \text { SGNF }>30 \& 90^{\circ} / \mathrm{s}, \& 90^{\circ} / \mathrm{s} \text { SGNF } \\
>30^{\circ} / \mathrm{s} \text { for individuals with \& without knee OA. } \\
\text { Reliability: test-retest knee extensor strength (ICC = } \\
0.83 \text { to } 0.95 \text { ). }\end{array}$ \\
\hline $\begin{array}{l}\text { White et al. } \\
(2010)[131]\end{array}$ & $\begin{array}{l}\text { Americans with knee OA or } \\
\text { at risk for knee OA }(n= \\
1801 ; \text { mean age }=62.7 \mathrm{y})\end{array}$ & $\begin{array}{l}\text { Cybex } 350 \text { dynamometer measured } \\
\text { knee extensor strength (PT) @ 60\% }\end{array}$ & $\begin{array}{l}\text { Responsiveness: reaching MCID in WOMAC physical } \\
\text { function @ follow-up was associated with } \downarrow \text { radiographic } \\
\text { knee OA, medication use, BMI, pain, \& depressive } \\
\text { symptoms \& } \uparrow \text { walking speed \& knee extensor strength } \\
@ \text { baseline. }\end{array}$ \\
\hline $\begin{array}{l}\text { Wu et al. } \\
(2008)[132]\end{array}$ & $\begin{array}{l}\text { Taiwanese with knee OA \& } \\
\text { normal }(n=11 ; \text { mean age } \\
=61.1 \text { y) or abnormal } \\
\text { patellar alignment }(n=14 ; \\
\text { mean age }=63.4 \mathrm{y}), \& \\
\text { healthy controls }(n=10 ; \\
\text { mean age }=61.4 \mathrm{y})\end{array}$ & $\begin{array}{l}\text { Cybex Norm dynamometer measured } \\
\text { knee extensor strength (body weight } \\
\text { adjusted PT) @ 80,120, \& } 240^{\circ} \% \text { s. }\end{array}$ & $\begin{array}{l}\text { Validity: knee extensor strength in groups with knee OA } \\
\text { SGNF }<\text { control, no difference between knee OA groups } \\
\text { based on patellar alignment. }\end{array}$ \\
\hline $\begin{array}{l}\text { Wu et al. } \\
(2018)[133]\end{array}$ & $\begin{array}{l}\text { Taiwanese with bilateral } \\
\text { knee OA (mean age }= \\
63.3 \text { y) assigned to platelet } \\
\text { rich plasma injection }(n= \\
\text { 20) or control }(n=20)\end{array}$ & $\begin{array}{l}\text { Biodex System } 3 \text { measured concentric } \\
\text { isokinetic strength (PT) of knee } \\
\text { flexors \& extensors @ } 60 \& 180^{\circ} / \mathrm{s} \text {. }\end{array}$ & $\begin{array}{l}\text { Responsiveness: SGNF } \uparrow \text { in all strength @ } 60^{\circ} / \mathrm{s} @ 2 \\
\text { week through } 6 \text { months post injection in both groups, } \\
\text { although no difference between groups. }\end{array}$ \\
\hline $\begin{array}{l}\text { Yázigi } \\
(2018)[134]\end{array}$ & $\begin{array}{l}\text { Portuguese with obesity \& } \\
\text { knee OA }(n=48 ; \text { mean age } \\
=55 \mathrm{y})\end{array}$ & $\begin{array}{l}\text { Biodex System } 3 \text { measured bilateral } \\
\text { isokinetic \& isometric knee flexor \& } \\
\text { extensor strength (bodyweight } \\
\text { adjusted PT). }\end{array}$ & $\begin{array}{l}\text { Validity: SGNF correlation between all strength } \\
\text { measures \& } 6 \text { minute Walk Test }(r=0.505 \text { to } 0.655) \text {. }\end{array}$ \\
\hline $\begin{array}{l}\text { Yilmaz et al. } \\
(2010)[135]\end{array}$ & $\begin{array}{l}\text { Turks with knee OA } \\
\text { assigned strength training } \\
\text { with EMG-biofeedback ( } n \\
=20 ; \text { mean age }=55.6 \text { y) or } \\
\text { strength training without } \\
\text { EMG-biofeedback ( } n=20 \text {; } \\
59.4 \text { y). }\end{array}$ & $\begin{array}{l}\text { Cybex dynamometer measured knee } \\
\text { flexor \& extensor strength (PT) @ } 60 \\
\& 180^{\circ} / \mathrm{s}, \& \text { isometric knee extensor } \\
\text { strength (PT) @ } 65^{\circ} .\end{array}$ & $\begin{array}{l}\text { Responsiveness: SGNF } \uparrow \text { in all knee strength measures } \\
\text { in both groups, no between group differences. }\end{array}$ \\
\hline
\end{tabular}


Table 1, continued

\begin{tabular}{|c|c|c|c|}
\hline $\begin{array}{l}\text { Authorship } \\
\text { (year) }\end{array}$ & Participants & Isokinetic procedures & Findings \\
\hline $\begin{array}{l}\text { Yoon et al. } \\
\text { (2018) [136] }\end{array}$ & $\begin{array}{l}\text { Japanese females with knee } \\
\text { OA assigned whole body } \\
\text { vibration therapy } \& \text { maslinic } \\
\text { acid supplementation }(n= \\
11 \text {; mean age }=69.5 \text { y) or } \\
\text { whole body vibration } \\
\text { therapy } \& \text { placebo }(n=15 ; \\
\text { mean age }=71.0 \mathrm{y})\end{array}$ & $\begin{array}{l}\text { Biodex System } 4 \text { measured maximal } \\
\text { isometric knee extensor strength } \\
\text { (bodyweight adjusted PT) @ } 60^{\circ} \text { of } \\
\text { flexion, \& isokinetic knee flexion \& } \\
\text { extension strength (bodyweight } \\
\text { adjusted PT) @ } 60^{\circ} / \mathrm{s} \text {. }\end{array}$ & $\begin{array}{l}\text { Responsiveness: knee with Kellgren-Lawrence grade } \leqslant \\
2 \text { had SGNF } \uparrow \text { in strength measures for both groups. } \\
\text { Knees with Kellgren-Lawrence grade } \geqslant 3 \text { had SGNF } \uparrow \\
\text { for both groups on isokinetic flexor strength \& only } \\
\text { masclinic acid group for extensor strength. }\end{array}$ \\
\hline
\end{tabular}

${ }^{*} \mathrm{OA}=$ osteoarthritis, $\mathrm{PT}=$ peak torque, $\mathrm{SGNF}=$ significant/significantly, $\mathrm{BMI}=$ body mass index, $\mathrm{ICC}=$ intraclass correlation coefficient, $\mathrm{MDC}=$ minimal detectable change, $\mathrm{OR}=$ odds ratio, $r=$ Pearson correlation, $\beta=$ standardized regression coefficient, $r^{2}=$ variance, $\mathrm{RR}=$ relative risk, KOOS = Knee Injury Osteoarthritis Outcome Score, WOMAC = Western Ontario McMaster Universities Osteoarthritis Index, TKA $=$ total knee arthroplasty.

vant information from these articles are summarized in Table 1 [8-136].

The summarized information demonstrates that isokinetic dynamometry is frequently utilized to measure strength of the knee flexor and extensor muscles in the presence of OA. Testing has been reported throughout the world with Asia, Europe, North America, and South America producing the bulk of published work. Various dynamometers have been used although Biodex, Cybex, En-Knee, and Kin-Com are most frequently reported. Most protocols have measured concentric contractions, with a primary outcome of peak torque (PT) or PT adjusted for body weight while fewer studies have reported work, power, fatigue, and agonist/antagonist ratio. Isokinetic test velocities range from 30 to $240 \%$, with most studies utilizing $60^{\circ} / \mathrm{s}$ - which appears to produce larger PT values than higher or lower velocities $[12,72,130]$.

Isokinetic knee strength measurements have demonstrated convergent validity with several related factors. Knee flexor and extensor strength have been shown to be strongly correlated ( $r=0.79$ to 0.97 ) $[61,67,74,123$, 124], with knee extensor testing producing larger torque values than flexor testing [72]. Concentric and eccentric measurements have been reported to be highly correlated ( $r=0.61$ to 0.88 ), eccentric efforts demonstrating larger torque values than concentric efforts for both the knee flexor and extensor muscles [53]. A strong correlation exists between the right and left lower extremities for knee flexor and extensor ( $r=0.80$ to 0.90$)$ strength [46,124]. Isokinetic strength has demonstrated strong correlations between testing velocities for the knee extensors [20]. Muscle cross-sectional area and isokinetic strength ( $r=0.49$ to 0.78 ) have been found to be positively correlated with cross-sectional areapartially explaining the variability in isokinetic strength performance [53]. Body mass index (BMI) may or may not be associated with lower knee strength in the presence of knee OA [65,108].

The clinimetric properties of isokinetic knee strength measurement have been supported via known groups or conditions validity in various ways. Dominant limb strength is larger than non-dominant limb strength [79] and strength measurements in the lower extremity affected with knee OA tend to be lower than in the unaffected limb $[64,67,122,130]$ but no difference has also been reported [87]. Knee extensor strength tends to be greater in individuals without knee OA than with knee OA [8], younger adults have greater strength than older adults $[8,66,88]$, and middle-aged adults with knee OA have similar strength to older adults without knee OA [8]. Knee flexor and extensor strength also tends to be greater in males than females $[18,50,51,61,105,107]$. Body weight is inversely correlated with knee extensor strength more frequently in females than males $[29,115]$, and those who develop incident knee OA [115].

Healthy adults tend to have stronger knee flexors [23, $30,33,45,48,79,115,117]$ and extensors [19,21,23,30, $33,38,45,48,66,79,82,91,110,114,115,117,132]$ than individuals with knee OA, however some studies have reported no difference in the flexors $[30,66]$ and extensors $[38,120]$ based upon presence or absence of knee OA. Knee flexor [19,33,45] and extensor [20,33,45] strength tends to be larger in the early stages of OA than later in the natural course, although some studies have reported no strength differences based upon disease severity [29,51]. Isometric knee strength is reportedly lower in the presence of knee OA [21,25,60,117] and worsens with disease severity [66] with few exceptions [20,91]. A correlation appears to exist between knee cartilage integrity and isometric knee flexor and extensor strength [66,121], but not isokinetic strength [121]. The variability in knee extensor isokinetic and isometric strength may be partially 
explained by patellofemoral cartilage integrity, extent of cartilage lesions, loose bodies, synovitis or effusion, and pain [20]. Significantly higher medial and lateral patellofemoral joint cartilage damage and lateral patellofemoral bone marrow lesions have been found in those in the lowest tertile of knee extensor strength [116].

Pain appears to be negatively correlated with knee strength $(r=-0.33$ to -0.22$)$ [51] with individuals experiencing knee pain demonstrating lower strength than pain free controls or pain free knee OA participants $[30,38,63,82,114]$. An inverse correlation between pain catastrophizing and isometric knee flexor strength has been reported [21]. Muscle strength may not contribute to pain variability [75]. An inverse correlation of pain catastrophizing with isometric knee flexor strength and kinesiophobia with isometric knee extensor strength have been reported [21]. Functional self-efficacy has demonstrated a correlation with knee flexor and extensor strength, with the variability in self-efficacy partial explained by knee stiffness, flexor strength, age, and depression [74].

Functional measurements have been associated with knee strength measurement utilizing isokinetic dynamometers. Knee strength has been identified as inversely associated with functional ambulation measures including the Get Up and Go Test [13,46,102,124,126], Timed Up and Go Test [26,49], 6 minute Walk Test [49, 134], 100-meter walk test [123,124], 20-meter walk test [18], gait speed [92,93,122], and walking time [67]. Difficulty rising from a chair has been associated with lower knee extensor strength in females [18]. Functional performance on the 30-second Chair Stand Test has been correlated with knee flexor and extensor strength ( $r=0.51$ to 0.56 ) [49]. Stair ambulation time tends to be inversely correlated to knee strength [26,46,49, $53,67,102,122]$. Knee extensor strength has been associated with lower levels of impairment on the Knee Injury and Osteoarthritis Outcome Score (KOOS) [94], Western Ontario McMaster Universities Osteoarthritis Index (WOMAC) [13,14,51,102,109,123,124,126] and Short Form-36 [92] physical function subscales. There may [40] or may not $[63,88]$ be an association between knee strength and proprioception. An association between knee strength and balance may also be present $[61,63]$.

Other contributing factors association with knee strength have been reported in patients with knee OA. An inverse correlation may be present between markers of systemic inflammation [40,103] or vitamin D levels [65] and knee strength in participants with knee
OA [40,103]. Knee strength appears to be negatively influenced by comorbidity [51] and sleep apnea [112]. A history of falling may [41] or may not [10] be associated with lower knee strength values. Levinger et al. [69] have reported fewer steps after an induced forward fall in individuals with higher knee extensor strength performance.

The relative reliability of isokinetic dynamometry in measuring knee strength in the presence of knee OA has been characterized using intraclass correlation coefficients (ICCs). Test-retest reliability for knee isokinetic strength measurement has been noted as strong with ICCs $\geqslant 0.72$ in all studies $[11,13,17,19,20,29$, $32,53,62,72,106,107,116,130]$ except that of Carpenter et al. [32], who reported a range of ICCs from 0.60 to 0.95 . Despite strong test-retest reliability, strength values may significantly increase in novice individuals after familiarization with isokinetic measurement suggesting a learning effect [95]. Minimal detectable change (MDC) values have been reported for concentric knee extensor strength PT (33.9 Nm) [62] and body weight adjusted PT for concentric knee extensor $(0.03$ to $0.05 \mathrm{Nm} / \mathrm{kg}$ ) and eccentric knee extensor strength $=$ 0.14 to $0.19 \mathrm{Nm} / \mathrm{kg}$ [17].

Numerous studies have documented significant increases in knee strength after exercise interventions $[11,15,16,27,42,43,47,52,54-60,64,73,78,80,82$, $85,87,89,90,96-98,121,129,135]$ including strength/power training [27,47,55,56,80,82,85,89,90,121,135], isokinetic exercise $[47,52,57-59,64,73,78,98,129]$, aerobic exercise $[11,80,87,90]$, stretching $[85,90]$, kinesthesia and balance exercises [43], physical therapy/ physiotherapy [24,60,97], hydro/aquatic therapy [42, 54], Baduanjin [15,16], and exercise education [96]. Two studies reported no difference in knee strength subsequent to physiotherapy [28] or aquatic or land-based exercise [70] both however conflicting with the positive effects of exercise reported in the majority of studies.

Thermal modalities including short wave diathermy [9,34], hot packs [34], laser [31], and ultrasound $[34,58,59]$ have reportedly been associated with additional improvements in knee strength over exercise interventions alone. Electric modalities including transcutaneous electric stimulation [37], neuromuscular electric stimulation [77,119], and biofeedback [135] have also been reported to produce improvements in knee strength when combined with exercise interventions. Whole body vibration training in combination with exercise may [27] or may not [85] produce larger increases in knee strength than exercise training alone, or maslinic acid supplementation [136]. 
Kinesiotape tends to produce no difference in knee strength compared with sham taping [84,128]; conflicting results have been reported relative to control applications [17]. Although sparsely reported, knee extensor strength may improve with application of a magnetic knee wrap [35] or knee brace [76]. Intraarticular hyaluronic acid injections tend to increase knee strength measures $[44,58,83,118]$ with one study reporting no difference [24]. Wu et al. [133] reported increased knee strength after platelet rich plasma injections although no difference was identified compared to placebo injection.

The available evidence of isokinetic measurement of knee strength the presence of knee OA is extensive, dating to at least the work of Lankhorst et al. [67] in 1985. Numerous studies have evaluated the association of knee strength and the presence and severity of knee $\mathrm{OA}$, age, gender, and body type. Isokinetic dynamometry has been shown to be a valid and reliable measure of knee flexor and extensor muscle strength. In the presence of knee OA, change in knee strength over time and with various interventions have been reported.

This review utilized only one database (PubMed) which may have limited the pool of available articles meeting the search and inclusion criteria. It is unlikely, however, that the inclusion of additional databases would substantially alter the summary provided in this review.

\section{Conclusion}

Isokinetic measurement of knee strength is wellsupported for individuals with knee OA. The evidence supports utilization of isokinetic measurement for the identification of strength impairments and subsequent responsiveness to therapeutic interventions.

\section{Conflict of interest}

The author declares no conflict of interest.

\section{References}

[1] Vos T, Flaxman AD, Naghavi M, Lozano R, Michaud C, Ezzati M, et al. Years lived with disability (YLDs) for 1160 sequelae of 289 diseases and injuries 1990-2010: A systematic analysis for the Global Burden of Disease Study 2010. Lancet 2012; 380: 2163-2196.

[2] Palazzo C, Nguyen C, Lefevre-Colau M, Rannou F, Poiraudeau S. Risk factors and burden of osteoarthritis. Ann Phys Rehabil Med 2016; 59: 134-138.
[3] Gay C, Guiguet-Auclair C, Mourgues C, Gerbaud L, Coudeyre E. Physical activity level and association with behavioral factors in knee osteoarthritis. Ann Phys Rehabil Med 2019; 62: 14-20.

[4] Nguyen C, Lefèvre-Colau M, Poiraudeau S, Rannou F. Rehabilitation (exercise and strength training) and osteoarthritis: A critical narrative review. Ann Phys Rehabil Med 2016; 59: 190-195.

[5] Bannuru RR, Osani MC, Vaysbrot EE, Arden NK, Bennell K, Bierma-Zeinstra S, et al. OARSI guidelines for the non-surgical management of knee, hip, and polyarticular osteoarthritis. Osteoarthritis Cartilage 2019; 27: 1578-1589.

[6] Fransen M, McConnell S, Harmer AR, Van der Esch M, Simic M, Bennell KL. Exercise for osteoarthritis of the knee: A Cochrane systematic review. Br J Sports Med 2015; 49: 1554-1557.

[7] Coudeyre E, Jegu AG, Giustanini M, Marrel JP, Edouard $\mathrm{P}$, Pereira B. Isokinetic muscle strengthening for knee osteoarthritis: A systematic review of randomized controlled trials with meta-analysis. Ann Phys Rehabil Med 2016; 59: 207-215.

[8] Aily JB, de Noronha M, de Almeida AC, Pedroso MG, Maciel JG, Mattiello-Sverzut AC, et al. Evaluation of vastus lateralis architecture and strength of knee extensors in middle-aged and older individuals with knee osteoarthritis. Clin Rheumatol 2019; 38: 2603-2611.

[9] Akyol Y, Durmus D, Alayli G, Tander B, Bek Y, Canturk F, et al. Does short-wave diathermy increase the effectiveness of isokinetic exercise on pain, function, knee muscle strength, quality of life, and depression in the patients with knee osteoarthritis? A randomized controlled clinical study. Eur J Phys Rehabil Med 2010; 46: 325-336.

[10] Alencar MA, Arantes PM, Dias JM, Kirkwood RN, Pereira LS, Dias RC. Muscular function and functional mobility of faller and non-faller elderly women with osteoarthritis of the knee. Braz J Med Biol Res 2007; 40: 277-283.

[11] Alkatan M, Baker JR, Machin DR, Park W, Akkari AS, Pasha $\mathrm{EP}$, et al. Improved function and reduced pain after swimming and cycling training in patients with osteoarthritis. J Rheumatol 2016; 43(3): 666-672.

[12] Almosnino S, Brandon SC, Sled EA. Does choice of angular velocity affect pain level during isokinetic strength testing of knee osteoarthritis patients? Eur J Phys Rehabil Med 2012; 48: 569-575.

[13] Altubasi IM. Knee joint laxity does not moderate the relationship between quadriceps strength and physical function in knee osteoarthritis patients: A cross-sectional study. Knee 2018; 25: 699-703.

[14] Amin S, Baker K, Niu J, Clancy M, Goggins J, Guermazi A, et al. Quadriceps strength and the risk of cartilage loss and symptom progression in knee osteoarthritis. Arthritis Rheum 2009; 60: 189-198.

[15] An B, Dai K, Zhu Z, Wang Y, Hao Y, Tang T, et al. Baduanjin alleviates the symptoms of knee osteoarthritis. J Altern Complement Med 2008; 14: 167-174.

[16] An BC, Wang Y, Jiang X, Lu HS, Fang ZY, Wang Y, et al. Effects of Baduanjin exercise on knee osteoarthritis: A one-year study. Chin J Integr Med 2013; 19: 143-148.

[17] Anandkumar S, Sudarshan S, Nagpal P. Efficacy of kinesio taping on isokinetic quadriceps torque in knee osteoarthritis: A double blinded randomized controlled study. Physiother Theory Pract 2014; 30: 375-383.

[18] Bacon KL, Segal NA, Oiestad BE, Lewis CE, Nevitt MC, Brown $\mathrm{C}$, et al. Thresholds in the relationship of quadriceps 
strength with functional limitations in women with knee osteoarthritis. Arthritis Care Res 2018; 71: 1186-1193.

[19] Baert IA, Mahmoudian A, Nieuwenhuys A, Jonkers I, Staes F, Luyten FP, et al. Proprioceptive accuracy in women with early and established knee osteoarthritis and its relation to functional ability, postural control, and muscle strength. Clin Rheumatol 2013; 32: 1365-1374

[20] Baert IA, Staes F, Truijen S, Mahmoudian A, Noppe N, Vanderschueren $\mathrm{G}$, et al. Weak associations between structural changes on MRI and symptoms, function and muscle strength in relation to knee osteoarthritis. Knee Surg Sports Traumatol Arthrosc 2014; 22: 2013-2025.

[21] Baert IAC, Meeus M, Mahmoudian A, Luyten FP, Nijs J, Verschueren SMP. Do psychosocial factors predict muscle strength, pain, or physical performance in patients with knee osteoarthritis? J Clin Rheumatol 2017; 23: 308-316

[22] Barker T, Rogers VE, Henriksen VT, Dixon BM, Momberger NG, Rasmussen GL, et al. Muscular-based and patientreported outcomes differentially associate with circulating superoxide dismutases and cytokines in knee osteoarthritis. Cytokine 2019; 115: 45-49.

[23] Batista JP, Facci LM, Pelegrinelli ARM, von Werder SCFA, Souza DC, Taglietti M, et al. Joint angle and movement velocity effects on muscle activity of elderly with knee osteoarthritis - Categorized and probabilistic analysis. J Electromyogr Kinesiol 2018; 41: 50-59.

[24] Bayramoglu M, Karatas M, Cetin N, Akman N, Sozay S, Dilek A. Comparison of two different viscosupplements in knee osteoarthritis - a pilot study. Clin Rheumatol 2003; 22 118-122.

[25] Bayramoglu M, Toprak R, Sozay S. Effects of osteoarthritis and fatigue on proprioception of the knee joint. Arch Phys Med Rehabil 2007; 88: 346-350.

[26] Bily W, Sarabon N, Lofler S, Franz C, Wakolbinger R, Kern H. Relationship between strength parameters and functional performance tests in patients with severe knee osteoarthritis. PM R 2019; 11: 834-842.

[27] Bokaeian HR, Bakhtiary AH, Mirmohammadkhani M, Moghimi J. The effect of adding whole body vibration training to strengthening training in the treatment of knee osteoarthritis: A randomized clinical trial. J Bodyw Mov Ther 2016; 20: 334-340

[28] Borjesson M, Robertson E, Weidenhielm L, Mattsson E, Olsson E. Physiotherapy in knee osteoarthrosis: Effect on pain and walking. Physiother Res Int 1996; 1: 89-97.

[29] Brandt KD, Heilman DK, Slemenda C, Katz BP, Mazzuca SA, Braunstein EM, et al. Quadriceps strength in women with radiographically progressive osteoarthritis of the knee and those with stable radiographic changes. J Rheumatol 1999; 26: 2431-2437.

[30] Brandt KD, Heilman DK, Slemenda C, Katz BP, Mazzuca S, Braunstein EM, et al. A comparison of lower extremity muscle strength, obesity, and depression scores in elderly subjects with knee pain with and without radiographic evidence of knee osteoarthritis. J Rheumatol 2000; 27: 1937-1946.

[31] Bulow PM, Jensen H, Danneskiold-Samsoe B. Low power Ga-Al-As laser treatment of painful osteoarthritis of the knee. A double-blind placebo-controlled study. Scand J Rehabil Med 1994; 26: 155-159.

[32] Carpenter MR, Carpenter RL, Peel J, Zukley LM, Angelopoulou KM, Fischer I, et al. The reliability of isokinetic and isometric leg strength measures among individuals with symptoms of mild osteoarthritis. J Sports Med Phys Fitness 2006; 46: 585-589.
[33] Cavanellas NT, Cossich VRA, Nicoliche EB, Martins MB, Sousa EB, Salles JI. Comparative analysis of quadriceps and hamstrings strength in knee osteoarthritis before and after total knee arthroplasty: A cross-sectional study. Rev Bras Ortop 2018; 53: 158-164

[34] Cetin N, Aytar A, Atalay A, Akman MN. Comparing hot pack, short-wave diathermy, ultrasound, and TENS on isokinetic strength, pain, and functional status of women with osteoarthritic knees: A single-blind, randomized, controlled trial. Am J Phys Med Rehabil 2008; 87: 443-451.

[35] Chen CY, Chen CL, Hsu SC, Chou SW, Wang KC. Effect of magnetic knee wrap on quadriceps strength in patients with symptomatic knee osteoarthritis. Arch Phys Med Rehabil 2008; 89: 2258-2264.

[36] Cherian JJ, McElroy MJ, Kapadia BH, Bhave A, Mont MA. Prospective case series of NMES for quadriceps weakness and decrease function in patients with osteoarthritis of the knee. J Long Term Eff Med Implants 2015; 25: 301-306.

[37] Cherian JJ, Kapadia BH, Bhave A, McElroy MJ, Cherian C, Harwin SF, et al. Use of transcutaneous electrical nerve stimulation device in early osteoarthritis of the knee. J Knee Surg 2015; 28: 321-327.

[38] Conroy MB, Kwoh CK, Krishnan E, Nevitt MC, Boudreau $\mathrm{R}$, Carbone LD, et al. Muscle strength, mass, and quality in older men and women with knee osteoarthritis. Arthritis Care Res 2012; 64: 15-21.

[39] Cornish SM, Peeler JD. No effect of creatine monohydrate supplementation on inflammatory and cartilage degradation biomarkers in individuals with knee osteoarthritis. Nutr Res 2018; 51: 57-66.

[40] Cudejko T, van der Esch M, van der Leeden M, Holla J, Roorda LD, Lems W, et al. Proprioception mediates the association between systemic inflammation and muscle weakness in patients with knee osteoarthritis: Results from the Amsterdam Osteoarthritis cohort. J Rehabil Med 2018; 50: 67-72.

[41] de Zwart AH, van der Esch M, Pijnappels MA, Hoozemans MJ, van der Leeden M, Roorda LD, et al. Falls associated with muscle strength in patients with Knee osteoarthritis and self-reported knee instability. J Rheumatol 2015; 42: 1218 1223.

[42] Dias JM, Cisneros L, Dias R, Fritsch C, Gomes W, Pereira $\mathrm{L}$, et al. Hydrotherapy improves pain and function in older women with knee osteoarthritis: A randomized controlled trial. Braz J Phys Ther 2017; 21: 449-456.

[43] Diracoglu D, Aydin R, Baskent A, Celik A. Effects of kinesthesia and balance exercises in knee osteoarthritis. J Clin Rheumatol 2005; 11: 303-310.

[44] Diracoglu D, Vural M, Baskent A, Dikici F, Aksoy C. The effect of viscosupplementation on neuromuscular control of the knee in patients with osteoarthritis. J Back Musculoskelet Rehabil 2009; 22: 1-9.

[45] Diracoglu D, Baskent A, Yagci I, Ozcakar L, Aydin R. Isokinetic strength measurements in early knee osteoarthritis. Acta Reumatol Port 2009; 34: 72-77.

[46] Edelaar LM, van Dieen JH, van der Esch M, Roorda LD, Dekker J, Lems WF, et al. Nonlinear relationship between isokinetic muscle strength and activity limitations in patients with knee osteoarthritis: Results of the AmsterdamOsteoarthritis cohort. J Rehabil Med 2017; 49: 598-605.

[47] Eyigor S, Hepguler S, Capaci K. A comparison of muscle training methods in patients with knee osteoarthritis. Clin Rheumatol 2004; 23: 109-115.

[48] Germanou EI, Chatzinikolaou A, Malliou P, Beneka A, Jamurtas AZ, Bikos $\mathrm{C}$, et al. Oxidative stress and inflamma- 
tory responses following an acute bout of isokinetic exercise in obese women with knee osteoarthritis. Knee 2013; 20 : 581-590.

[49] Gkrilias P, Tsepis E, Kokkalis Z, Panagiotopoulos E, Megas $P$. The relationship between isokinetic strength and functional performance tests in patients with knee osteoarthritis. J Phys Ther Sci 2018; 30: 888-891.

[50] Glass NA, Torner JC, Frey Law LA, Wang K, Yang T, Nevitt $\mathrm{MC}$, et al. The relationship between quadriceps muscle weakness and worsening of knee pain in the MOST cohort: A 5-year longitudinal study. Osteoarthritis Cartilage 2013; 21: 1154-1159.

[51] Gokcen N, Basaran S, Benlidayi IC, Ozdemir C, Seydaoglu G. Which predicts quadriceps muscle strength in knee osteoarthritis: Biological markers or clinical variables? Arch Rheumatol 2016; 32: 32-38.

[52] Gur H, Cakin N, Akova B, Okay E, Kucukoglu S. Concentric versus combined concentric-eccentric isokinetic training: effects on functional capacity and symptoms in patients with osteoarthrosis of the knee. Arch Phys Med Rehabil 2002; 83 308-316.

[53] Gur H, Cakin N. Muscle mass, isokinetic torque, and functional capacity in women with osteoarthritis of the knee. Arch Phys Med Rehabil 2003; 84: 1534-1541.

[54] Ha GC, Yoon JR, Yoo CG, Kang SJ, Ko KJ. Effects of 12week aquatic exercise on cardiorespiratory fitness, knee isokinetic function, and Western Ontario and McMaster University osteoarthritis index in patients with knee osteoarthritis women. J Exerc Rehabil 2018; 14: 870-876.

[55] Hall M, Hinman RS, Wrigley TV, Kasza J, Lim BW, Bennell KL. Knee extensor strength gains mediate symptom improvement in knee osteoarthritis: Secondary analysis of a randomised controlled trial. Osteoarthritis Cartilage 2018; 26: 495-500.

[56] Harper SA, Roberts LM, Layne AS, Jaeger BC, Gardner AK, Sibille KT, et al. Blood-flow restriction resistance exercise for older adults with knee osteoarthritis: A pilot randomized clinical trial. J Clin Med 2019; 8: 265.

[57] Huang MH, Lin YS, Yang RC, Lee CL. A comparison of various therapeutic exercises on the functional status of patients with knee osteoarthritis. Semin Arthritis Rheum 2003; 32 398-406.

[58] Huang MH, Yang RC, Lee CL, Chen TW, Wang MC. Preliminary results of integrated therapy for patients with knee osteoarthritis. Arthritis Rheum 2005; 53: 812-820.

[59] Huang MH, Lin YS, Lee CL, Yang RC. Use of ultrasound to increase effectiveness of isokinetic exercise for knee osteoarthritis. Arch Phys Med Rehabil 2005; 86: 1545-1551.

[60] Hurley MV, Newham DJ. The influence of arthrogenous muscle inhibition on quadriceps rehabilitation of patients with early, unilateral osteoarthritic knees. Br J Rheumatol 1993; 32: 127-131.

[61] Jadelis K, Miller ME, Ettinger WH, Jr, Messier SP. Strength, balance, and the modifying effects of obesity and knee pain: Results from the Observational Arthritis Study in Seniors (oasis). J Am Geriatr Soc 2001; 49: 884-891.

[62] Kean CO, Birmingham TB, Garland SJ, Bryant DM, Giffin JR. Minimal detectable change in quadriceps strength and voluntary muscle activation in patients with knee osteoarthritis. Arch Phys Med Rehabil 2010; 91: 1447-1451.

[63] Kim D, Park G, Kuo LT, Park W. The effects of pain on quadriceps strength, joint proprioception and dynamic balance among women aged 65 to 75 years with knee osteoarthritis. BMC Geriatr 2018; 18: 245.
[64] King LK, Birmingham TB, Kean CO, Jones IC, Bryant DM, Giffin JR. Resistance training for medial compartment knee osteoarthritis and malalignment. Med Sci Sports Exerc 2008; 40: 1376-1384

[65] Koeckhoven E, van der Leeden M, Roorda LD, van Schoor NM, Lips P, de Zwart A, et al. The association between serum 25-hydroxy vitamin D level and upper leg strength in patients with knee osteoarthritis: Results of the Amsterdam Osteoarthritis Cohort. J Rheumatol 2016; 43: 1400-1405.

[66] Kumar D, Karampinos DC, MacLeod TD, Lin W, Nardo L, Li X, et al. Quadriceps intramuscular fat fraction rather than muscle size is associated with knee osteoarthritis. Osteoarthritis Cartilage 2014; 22: 226-234.

[67] Lankhorst GJ, Van de Stadt RJ, Van der Korst JK. The relationships of functional capacity, pain, and isometric and isokinetic torque in osteoarthrosis of the knee. Scand J Rehabil Med 1985; 17: 167-172.

[68] Lee SH, Lee JH, Ahn SE, Park MJ, Lee DH. Correlation between quadriceps endurance and adduction moment in medial knee osteoarthritis. PLoS One 2015; 10: e0141972.

[69] Levinger P, Downie C, Nagano H, Petersen A, Hayes A, Sanders KM, et al. Stepping strategy used to recover balance during an induced fall is associated with impaired function and strength in people with knee osteoarthritis. Int J Rheum Dis 2018; 21: 1763-1771.

[70] Lim JY, Tchai E, Jang SN. Effectiveness of aquatic exercise for obese patients with knee osteoarthritis: a randomized controlled trial. PM R 2010; 2: 723-31.

[71] Lim SH, Hong BY, Oh JH, Lee JI. Relationship between knee alignment and the electromyographic activity of quadriceps muscles in patients with knee osteoarthritis. J Phys Ther Sci 2015; 27: 1261-1265.

[72] Madsen OR, Brot C. Assessment of extensor and flexor strength in the individual gonarthrotic patient: Interpretation of performance changes. Clin Rheumatol 1996; 15: 154-160.

[73] Malas FU, Ozcakar L, Kaymak B, Ulasli A, Guner S, Kara $\mathrm{M}$, et al. Effects of different strength training on muscle architecture: Clinical and ultrasonographic evaluation in knee osteoarthritis. PM R 2013; 5: 655-662.

[74] Maly MR, Costigan PA, Olney SJ. Determinants of self efficacy for physical tasks in people with knee osteoarthritis. Arthritis Rheum 2006; 55: 94-101.

[75] Maly MR, Costigan PA, Olney SJ. Mechanical factors relate to pain in knee osteoarthritis. Clin Biomech 2008; 23: 796805 .

[76] Matsuno H, Kadowaki KM, Tsuji H. Generation II knee bracing for severe medial compartment osteoarthritis of the knee. Arch Phys Med Rehabil 1997; 78: 745-749.

[77] Matsuse H, Hashida R, Takano Y, Omoto M, Nago T, Bekki $\mathrm{M}$, et al. Walking exercise simultaneously combined with neuromuscular electrical stimulation of antagonists resistance improved muscle strength, physical function, and knee pain in symptomatic knee osteoarthritis: A single-arm study. J Strength Cond Res 2017; 31: 171-180.

[78] Maurer BT, Stern AG, Kinossian B, Cook KD, Schumacher HR, Jr. Osteoarthritis of the knee: Isokinetic quadriceps exercise versus an educational intervention. Arch Phys Med Rehabil 1999; 80: 1293-1299.

[79] Messier SP, Loeser RF, Hoover JL, Semble EL, Wise CM. Osteoarthritis of the knee: Effects on gait, strength, and flexibility. Arch Phys Med Rehabil 1992; 73: 29-36.

[80] Messier SP, Loeser RF, Mitchell MN, Valle G, Morgan TP, Rejeski WJ, et al. Exercise and weight loss in obese older 
adults with knee osteoarthritis: A preliminary study. J Am Geriatr Soc 2000; 48: 1062-1072.

[81] Messier SP, Glasser JL, Ettinger WH, Jr, Craven TE, Miller ME. Declines in strength and balance in older adults with chronic knee pain: A 30-month longitudinal, observational study. Arthritis Rheum 2002; 47: 141-148.

[82] Mikesky AE, Mazzuca SA, Brandt KD, Perkins SM, Damush $T$, Lane KA. Effects of strength training on the incidence and progression of knee osteoarthritis. Arthritis Rheum 2006; 55 : 690-699.

[83] Miltner O, Schneider U, Siebert CH, Niedhart C, Niethard FU. Efficacy of intraarticular hyaluronic acid in patients with osteoarthritis-a prospective clinical trial. Osteoarthritis Cartilage 2002; 10: 680-686.

[84] Ogut H, Guler H, Yildizgoren MT, Velioglu O, Turhanoglu AD. Does kinesiology taping improve muscle strength and function in knee osteoarthritis? A single-blind, randomized and controlled study. Arch Rheumatol 2018; 33: 335-343

[85] Park YG, Kwon BS, Park JW, Cha DY, Nam KY, Sim KB, et al. Therapeutic effect of whole body vibration on chronic knee osteoarthritis. Ann Rehabil Med 2013; 37: 505-515.

[86] Patsika G, Kellis E, Kofotolis N, Salonikidis K, Amiridis IG. Synergetic and antagonist muscle strength and activity in women with knee osteoarthritis. J Geriatr Phys Ther 2014; 37: 17-23.

[87] Peeler J, Ripat J. The effect of low-load exercise on joint pain, function, and activities of daily living in patients with knee osteoarthritis. Knee 2018; 25: 135-145.

[88] Peixoto JG, Dias JM, Dias RC, da Fonseca ST, TeixeiraSalmela LF. Relationships between measures of muscular performance, proprioceptive acuity, and aging in elderly women with knee osteoarthritis. Arch Gerontol Geriatr 2011; 53: e253-7.

[89] Pelletier D, Gingras-Hill C, Boissy P. Power training in patients with knee osteoarthritis: A pilot study on feasibility and efficacy. Physiother Can 2013; 65: 176-182.

[90] Peloquin L, Bravo G, Gauthier P, Lacombe G, Billiard JS Effects of a cross-training exercise program in persons with osteoarthritis of the knee a randomized controlled trial. J Clin Rheumatol 1999; 5: 126-136.

[91] Petrella M, Gramani-Say K, Serrao PR, Lessi GC, Barela JA, Carvalho RP, et al. Measuring postural control during minisquat posture in men with early knee osteoarthritis. Hum Mov Sci 2017; 52: 108-116.

[92] Pua YH, Liang Z, Ong PH, Bryant AL, Lo NN, Clark RA. Associations of knee extensor strength and standing balance with physical function in knee osteoarthritis. Arthritis Care Res 2011; 63: 1706-1714.

[93] Pua YH, Clark RA, Ong PH, Bryant AL, Lo NN, Liang Z. Association between seated postural control and gait speed in knee osteoarthritis. Gait Posture 2013; 37: 413-418.

[94] Robbins SM, Birmingham TB, Callaghan JP, Jones GR, Chesworth BM, Maly MR. Association of pain with frequency and magnitude of knee loading in knee osteoarthritis. Arthritis Care Res 2011; 63: 991-997.

[95] Rodrigues-da-Silva JM, de Rezende MU, Spada TC, da Silva Francisco L, Greve JMD, Ciolac EG. Effects of motor learning on clinical isokinetic test performance in knee osteoarthritis patients. Clinics 2017; 72: 202-206

[96] Rodrigues da Silva JM, de Rezende MU, Spada TC, da Silva Francisco L, Dos Santos HP, de Andrade Souza R, et al. Muscular and functional capacity in subjects under treatment for knee osteoarthritis: Role of physical activity status. J Phys Act Health 2019; 16: 362-367.
[97] Rogind H, Bibow-Nielsen B, Jensen B, Moller HC, FrimodtMoller $\mathrm{H}$, Bliddal $\mathrm{H}$. The effects of a physical training program on patients with osteoarthritis of the knees. Arch Phys Med Rehabil 1998; 79: 1421-1427.

[98] Samut G, Dincer F, Ozdemir O. The effect of isokinetic and aerobic exercises on serum interleukin-6 and tumor necrosis factor alpha levels, pain, and functional activity in patients with knee osteoarthritis. Mod Rheumatol 2015; 25: 919-924.

[99] Sanchez-Ramirez DC, van der Leeden M, Knol DL, van der Esch M, Roorda LD, Verschueren S, et al. Association of postural control with muscle strength, proprioception, selfreported knee instability and activity limitations in patients with knee osteoarthritis. J Rehabil Med 2013; 45: 192-197.

[100] Sanchez-Ramirez DC, van der Leeden M, van der Esch M, Gerritsen M, Roorda LD, Verschueren S, et al. Association of serum C-reactive protein and erythrocyte sedimentation rate with muscle strength in patients with knee osteoarthritis. Rheumatology 2013; 52: 727-732.

[101] Sanchez-Ramirez DC, van der Leeden M, van der Esch M, Roorda LD, Verschueren S, van Dieen JH, et al. Elevated C-reactive protein is associated with lower increase in knee muscle strength in patients with knee osteoarthritis: A 2-year follow-up study in the Amsterdam Osteoarthritis (AMS-OA) cohort. Arthritis Res Ther 2014; 16: R123.

[102] Sanchez-Ramirez DC, van der Leeden M, van der Esch M, Roorda LD, Verschueren S, van Dieen J, et al. Increased knee muscle strength is associated with decreased activity limitations in established knee osteoarthritis: Two-year follow-up study in the Amsterdam osteoarthritis cohort. J Rehabil Med 2015; 47: 647-654.

[103] Santos ML, Gomes WF, Pereira DS, Oliveira DM, Dias JM, Ferrioli E, et al. Muscle strength, muscle balance, physical function and plasma interleukin-6 (IL-6) levels in elderly women with knee osteoarthritis (OA). Arch Gerontol Geriatr 2011; 52: 322-326.

[104] Schilke JM, Johnson GO, Housh TJ, O'Dell JR. Effects of muscle-strength training on the functional status of patients with osteoarthritis of the knee joint. Nurs Res 1996; 45: 6872.

[105] Segal NA, Torner JC, Felson D, Niu J, Sharma L, Lewis $\mathrm{CE}$, et al. Effect of thigh strength on incident radiographic and symptomatic knee osteoarthritis in a longitudinal cohort. Arthritis Rheum 2009; 61: 1210-1217.

[106] Segal NA, Torner JC, Felson DT, Niu J, Sharma L, Lewis $\mathrm{CE}$, et al. Knee extensor strength does not protect against incident knee symptoms at 30 months in the multicenter knee osteoarthritis (MOST) cohort. PM R 2009; 1: 459-465.

[107] Segal NA, Glass NA, Torner J, Yang M, Felson DT, Sharma L, et al. Quadriceps weakness predicts risk for knee joint space narrowing in women in the MOST cohort. Osteoarthritis Cartilage 2010; 18: 769-775.

[108] Segal NA, Zimmerman MB, Brubaker M, Torner JC. Obesity and knee osteoarthritis are not associated with impaired quadriceps specific strength in adults. PM R 2011; 3: 314-23.

[109] Serrao PR, Gramani-Say K, Lessi GC, Mattiello SM. Knee extensor torque of men with early degrees of osteoarthritis is associated with pain, stiffness and function. Rev Bras Fisioter 2012; 16: 289-294.

[110] Serrao PR, Vasilceac FA, Gramani-Say K, Lessi GC, Oliveira $\mathrm{AB}$, Reiff RB, et al. Men with early degrees of knee osteoarthritis present functional and morphological impairments of the quadriceps femoris muscle. Am J Phys Med Rehabil 2015; 94: 70-81. 
[111] Shakoor N, Felson DT, Niu J, Nguyen US, Segal NA, Singh $\mathrm{JA}$, et al. The association of vibratory perception and muscle strength with the incidence and worsening of knee instability: The Multicenter Osteoarthritis Study. Arthritis Rheumatol 2017; 69: 94-102.

[112] Silva A, Mello MT, Serrao PR, Luz RP, Ruiz F, Bittencourt $\mathrm{LR}$, et al. Influence of obstructive sleep apnea in the functional aspects of patients with osteoarthritis. J Clin Sleep Med 2018; 14: 265-270.

[113] Skou ST, Wise BL, Lewis CE, Felson D, Nevitt M, Segal NA, et al. Muscle strength, physical performance and physical activity as predictors of future knee replacement: A prospective cohort study. Osteoarthritis Cartilage 2016; 24: 1350-1356.

[114] Slemenda C, Brandt KD, Heilman DK, Mazzuca S, Braunstein EM, Katz BP, et al. Quadriceps weakness and osteoarthritis of the knee. Ann Intern Med 1997; 127: 97-104.

[115] Slemenda C, Heilman DK, Brandt KD, Katz BP, Mazzuca SA, Braunstein EM, et al. Reduced quadriceps strength relative to body weight: a risk factor for knee osteoarthritis in women? Arthritis Rheum 1998; 41: 1951-1959.

[116] Stefanik JJ, Guermazi A, Zhu Y, Zumwalt AC, Gross KD, Clancy M, et al. Quadriceps weakness, patella alta, and structural features of patellofemoral osteoarthritis. Arthritis Care Res 2011; 63: 1391-1397.

[117] Tan J, Balci N, Sepici V, Gener FA. Isokinetic and isometric strength in osteoarthrosis of the knee. A comparative study with healthy women. Am J Phys Med Rehabil 1995; 74: 364-369.

[118] Tang SF, Chen CP, Chen MJ, Hong WH, Yu TY, Tsai WC. Improvement of muscle strength in osteoarthritic knee patients after intraarticular knee injection of hyaluronan. Am J Phys Med Rehabil 2005; 84: 274-277.

[119] Tok F, Aydemir K, Peker F, Safaz I, Taskaynatan MA, Ozgul A. The effects of electrical stimulation combined with continuous passive motion versus isometric exercise on symptoms, functional capacity, quality of life and balance in knee osteoarthritis: Randomized clinical trial. Rheumatol Int 2011; 31: 177-181.

[120] Tuna S, Balci N. The relationship between radiological severity and functional status in patients with knee osteoarthritis. Clin Rheumatol 2014; 33: 667-670.

[121] Tuna S, Balci N, Ozcakar L. The relationship between femoral cartilage thickness and muscle strength in knee osteoarthritis. Clin Rheumatol 2016; 35: 2073-2077.

[122] Valtonen AM, Poyhonen T, Manninen M, Heinonen A, Sipila S. Knee extensor and flexor muscle power explains stair ascension time in patients with unilateral late-stage knee osteoarthritis: A cross-sectional study. Arch Phys Med Rehabil 2015; 96: 253-259.

[123] van der Esch M, Steultjens M, Knol DL, Dinant H, Dekker J. Joint laxity and the relationship between muscle strength and functional ability in patients with osteoarthritis of the knee. Arthritis Rheum 2006; 55: 953-959.
[124] van der Esch M, Steultjens M, Harlaar J, Knol D, Lems W, Dekker J. Joint proprioception, muscle strength, and functional ability in patients with osteoarthritis of the knee. Arthritis Rheum 2007; 57: 787-793.

[125] van der Esch M, Steultjens M, Harlaar J, Wolterbeek N, Knol DL, Dekker J. Knee varus-valgus motion during gait - a measure of joint stability in patients with osteoarthritis? Osteoarthritis Cartilage 2008; 16: 522-525.

[126] van der Esch M, Steultjens M, Harlaar J, Wolterbeek N, Knol D, Dekker J. Varus-valgus motion and functional ability in patients with knee osteoarthritis. Ann Rheum Dis 2008; 67: 471-477.

[127] van der Esch M, Knoop J, van der Leeden M, Voorneman R, Gerritsen M, Reiding D, et al. Self-reported knee instability and activity limitations in patients with knee osteoarthritis: Results of the Amsterdam osteoarthritis cohort. Clin Rheumatol 2012; 31: 1505-1510.

[128] Wageck B, Nunes GS, Bohlen NB, Santos GM, de Noronha M. Kinesio Taping does not improve the symptoms or function of older people with knee osteoarthritis: A randomised trial. J Physiother 2016; 62: 153-158.

[129] Weng MC, Lee CL, Chen CH, Hsu JJ, Lee WD, Huang MH, et al. Effects of different stretching techniques on the outcomes of isokinetic exercise in patients with knee osteoarthritis. Kaohsiung J Med Sci 2009; 25: 306-315.

[130] Wessel J. Isometric strength measurements of knee extensors in women with osteoarthritis of the knee. J Rheumatol 1996; 23: 328-331.

[131] White DK, Keysor JJ, Lavalley MP, Lewis CE, Torner JC, Nevitt MC, et al. Clinically important improvement in function is common in people with or at high risk of knee OA: The MOST study. J Rheumatol 2010; 37: 1244-1251.

[132] Wu SH, Chu NK, Liu YC, Chen CK, Tang SF, Cheng CK. Relationship between the EMG ratio of muscle activation and bony structure in osteoarthritic knee patients with and without patellar malalignment. J Rehabil Med 2008; 40: 381-386.

[133] Wu YT, Hsu KC, Li TY, Chang CK, Chen LC. Effects of platelet-rich plasma on pain and muscle strength in patients with knee osteoarthritis. Am J Phys Med Rehabil 2018; 97 : 248-254.

[134] Yazigi F, Espanha M, Marques A, Teles J, Teixeira P. Predictors of walking capacity in obese adults with knee osteoarthritis. Acta Reumatol Port 2018; 43: 256-263.

[135] Yilmaz OO, Senocak O, Sahin E, Baydar M, Gulbahar S, Bircan C, et al. Efficacy of EMG-biofeedback in knee osteoarthritis. Rheumatol Int 2010; 30: 887-892.

[136] Yoon J, Kanamori A, Fujii K, Isoda H, Okura T. Evaluation of maslinic acid with whole-body vibration training in elderly women with knee osteoarthritis. PLoS One 2018; 13: e0194572. 